\title{
European Respiratory Society guidelines for the management of adult bronchiectasis
}

\author{
Eva Polverino ${ }^{1}$, Pieter C. Goeminne ${ }^{2,3}$, Melissa J. McDonnell ${ }^{4,5,6}$, \\ Stefano Aliberti $\mathbb{1}^{7}$, Sara E. Marshall ${ }^{8}$, Michael R. Loebinger ${ }^{9}$, \\ Marlene Murris ${ }^{10}$, Rafael Cantón ${ }^{11}$, Antoni Torres ${ }^{12}$, Katerina Dimakou ${ }^{13}$, \\ Anthony De Soyza ${ }^{14,15}$, Adam T. Hill ${ }^{16}$, Charles S. Haworth ${ }^{17}$, \\ Montserrat Vendrell ${ }^{18}$, Felix C. Ringshausen ${ }^{19}$, Dragan Subotic ${ }^{20}$, \\ Robert Wilson ${ }^{9}$, Jordi Vilaró ${ }^{21}$, Bjorn Stallberg ${ }^{22}$, Tobias Welte $^{19}$, \\ Gernot Rohde ${ }^{23}$, Francesco Blasi ${ }^{7}$, Stuart Elborn ${ }^{9,24}$, Marta Almagro ${ }^{25}$, \\ Alan Timothy ${ }^{25}$, Thomas Ruddy ${ }^{25}$, Thomy Tonia ${ }^{26}$, David Rigau ${ }^{27}$ and \\ James D. Chalmers ${ }^{28}$
}

@ERSpublications

The publication of the first ERS guidelines for bronchiectasis http://ow.ly/wQSO30dU0nE

Cite this article as: Polverino E, Goeminne PC, McDonnell MJ, et al. European Respiratory Society guidelines for the management of adult bronchiectasis. Eur Respir J 2017; 50: 1700629 [https://doi.org/ 10.1183/13993003.00629-2017].

ABSTRACT Bronchiectasis in adults is a chronic disorder associated with poor quality of life and frequent exacerbations in many patients. There have been no previous international guidelines.

The European Respiratory Society guidelines for the management of adult bronchiectasis describe the appropriate investigation and treatment strategies determined by a systematic review of the literature.

A multidisciplinary group representing respiratory medicine, microbiology, physiotherapy, thoracic surgery, primary care, methodology and patients considered the most relevant clinical questions (for both clinicians and patients) related to management of bronchiectasis. Nine key clinical questions were generated and a systematic review was conducted to identify published systematic reviews, randomised clinical trials and observational studies that answered these questions. We used the GRADE approach to define the quality of the evidence and the level of recommendations. The resulting guideline addresses the investigation of underlying causes of bronchiectasis, treatment of exacerbations, pathogen eradication, long term antibiotic treatment, anti-inflammatories, mucoactive drugs, bronchodilators, surgical treatment and respiratory physiotherapy.

These recommendations can be used to benchmark quality of care for people with bronchiectasis across Europe and to improve outcomes.

This article has supplementary material available from erj.ersjournals.com

Received: March 262017 | Accepted after revision: July 102017

The guidelines published by the European Respiratory Society (ERS) incorporate data obtained from a comprehensive and systematic literature review of the most recent studies available at the time. Health professionals are encouraged to take the guidelines into account in their clinical practice. However, the recommendations issued by this guideline may not be appropriate for use in all situations. It is the individual responsibility of health professionals to consult other sources of relevant information, to make appropriate and accurate decisions in consideration of each patient's health condition and in consultation with that patient and the patient's caregiver where appropriate and/or necessary, and to verify rules and regulations applicable to drugs and devices at the time of prescription.

This document was endorsed by the ERS Executive Committee and by the European Society of Clinical Microbiology and Infectious Disease in August 2017.

Copyright @ERS 2017 
Affiliations: 'Servei de Pneumologia, Hospital Universitari Vall d'Hebron (HUVH), Institut de Recerca Vall d'Hebron (VHIR); Fundación Clínic, Hospital Clínic de Barcelona, Universitat de Barcelona, IDIBAPS, CIBERES Barcelona, Barcelona, Spain. ${ }^{2}$ Dept of Respiratory Medicine, AZ Nikolaas, Sint-Niklaas, Belgium. ${ }^{3}$ Dept of Respiratory Medicine, UZ Leuven, Leuven, Belgium. ${ }^{4}$ Dept of Respiratory Medicine, Galway University Hospitals, Galway, Ireland. ${ }^{5}$ Lung Biology Group, National University of Ireland, Galway, Ireland. ${ }^{6}$ Institute of Cell and Molecular Biology, Newcastle University, Newcastle upon Tyne, UK. ${ }^{7}$ Dept of Pathophysiology and Transplantation, Università degli Studi di Milano, Internal Medicine Department, Respiratory Unit and Adult Cystic Fibrosis Center Fondazione IRCCS Cà Granda Ospedale Maggiore Policlinico Milan, Milan, Italy. ${ }^{8}$ Dept of Clinical Research, Immunology and Physiological Sciences, Wellcome, London, UK. ${ }^{9}$ Host Defence Unit, Royal Brompton Hospital, Imperial College, London, UK. ${ }^{10}$ Service de Pneumologie, Hôpital Larrey, CHU de Toulouse, Toulouse, France. ${ }^{11}$ Servicio de Microbiología, Hospital Universitario Ramón y Cajal and Instituto Ramón y Cajal de Investigación Sanitaria (IRYCIS), Madrid, Spain. ${ }^{12}$ Servei de Pneumologia, Hospital Clínic de Barcelona, Universitat de Barcelona, IDIBAPS, CIBERES Barcelona, Barcelona, Spain. ${ }^{13} 5$ th Pulmonary Dept, "Sotiria" Chest Hospital, Athens, Greece. ${ }^{14}$ Institute of Cellular Medicine, Newcastle University, Newcastle upon Tyne, UK. ${ }^{15}$ Bronchiectasis Service, Freeman Hospital, Newcastle upon Tyne, UK. ${ }^{16}$ Dept of Respiratory Medicine, Royal Infirmary and University of Edinburgh, Edinburgh, UK. ${ }^{17}$ Cambridge Centre for Lung Infection, Papworth Hospital, Cambridge, UK. ${ }^{18}$ Bronchiectasis Group, Girona Biomedical Research Institute (IDIBGI), Dr Trueta University Hospital, Girona, Spain. ${ }^{19}$ Dept of Respiratory Medicine, Hannover Medical School, Member of the German Centre for Lung Research, Hannover, Germany. ${ }^{20} \mathrm{Clinic}$ for Thoracic Surgery - Clinical Centre of Serbia, University of Belgrade, Belgrade, Serbia. ${ }^{21}$ FCS Blanquerna. Physical Activity and Health Group, Universitat Ramon Llull, Barcelona, Spain. ${ }^{22}$ Dept of Public Health and Caring Science, Family Medicine and Preventive Medicine, Uppsala University, Uppsala, Sweden. ${ }^{23}$ Dept of Respiratory Medicine, Maastricht University Medical Center, Maastricht, The Netherlands. ${ }^{24}$ Queen's University Belfast, Belfast, UK. ${ }^{25}$ European Lung Foundation (ELF)/EMBARC bronchiectasis patient advisory group. ${ }^{26}$ Institute of Social and Preventive Medicine, University of Bern, Bern, Switzerland. ${ }^{27}$ Iberoamerican Cochrane Center, Barcelona, Spain. ${ }^{28}$ College of Medicine, University of Dundee, Ninewells Hospital and Medical School, Dundee, UK.

Correspondence: James D. Chalmers, Scottish Centre for Respiratory Research, Ninewells Hospital and Medical School, Dundee, DD1 9SY, UK. E-mail: jchalmersđdundee.ac.uk

\section{Scope and objectives}

This guideline provides evidence-based recommendations for the management of adult patients with bronchiectasis. It only applies to patients with clinically significant bronchiectasis, defined by the presence of both permanent bronchial dilatation on computed tomography (CT) scanning and the clinical syndrome of cough, sputum production and/or recurrent respiratory infections. Radiological bronchiectasis may be evident in healthy asymptomatic individuals, particularly in the elderly [1] or may occur, for example, due to traction in interstitial lung disease. Such radiological bronchiectasis without clinical symptoms are not addressed in this guideline. The following conditions are also excluded: cystic fibrosis bronchiectasis, which has a distinct pathophysiology and treatment pathway, children with bronchiectasis, treatment of primary immunodeficiencies and non-tuberculous mycobacteria (NTM), where disease specific therapy is indicated. The majority of these clinical issues are addressed in other guidelines.

This guideline document does not address clinical and radiological diagnosis of bronchiectasis but rather focuses on key questions in management. Areas such as smoking cessation, nutrition, influenza and pneumococcal vaccination among other aspects of general management are not specifically addressed in this document. Readers are referred to relevance guidelines and national policies. A guideline document cannot address the full complexity of a disease such as bronchiectasis, hence all recommendations should be interpreted taking into account the clinical circumstances and patients' perceptions, values and preferences.

Table 1 provides a framework to understand the recommendations made in this document $[2,3]$.

The target audience for this guideline are all stakeholders involved bronchiectasis care. This includes specialists in respiratory medicine, infectious diseases, clinical microbiology, general internists, specialists in thoracic surgery, primary care physicians, pharmacists, respiratory physiotherapists, specialist nurses, regulatory authorities, pharmaceutical companies and policy makers. The guideline is also to inform people with bronchiectasis to help them to discuss with their care teams and to access appropriate care.

\section{Introduction}

Bronchiectasis is a chronic respiratory disease characterised by a clinical syndrome of cough, sputum production and bronchial infection, and radiologically by abnormal and permanent dilatation of the bronchi. The objectives of treatment in bronchiectasis are to prevent exacerbations, reduce symptoms,

Support statement: The task force was funded by the European Respiratory Society. Funding information for this article has been deposited with the Crossref Funder Registry.

Conflict of interest: D. Rigau and T. Tonia act as methodologists for the European Respiratory Society. All other disclosures can be found alongside this article at erj.ersjournals.com 
TABLE 1 Understanding the recommendations made in this document

\begin{tabular}{ll} 
Target group & Strong recommendations \\
\hline Patients & $\begin{array}{c}\text { All or almost all informed people would choose the } \\
\text { recommended choice for or against an } \\
\text { intervention. }\end{array}$ \\
Clinicians & $\begin{array}{c}\text { Most patients should receive the recommended } \\
\text { course of action. }\end{array}$
\end{tabular}

Policy makers The recommendation can be adopted as a policy in most situations.

\section{Conditional (weak) recommendations}

Most informed people would choose the recommended course of action, but a substantial number would not.

Recognise that different choices will be appropriate for different patients. Clinicians and other healthcare providers need to devote more time to the process of shared decision making by which they ensure that the informed choice reflects individual values and preferences; decision aids and shared decision making are particularly useful.

Policy making will require substantial debate and involvement of many stakeholders.

\#: strong recommendations based on high quality evidence will apply to most patients for whom these recommendations are made, but they may not apply to all patients in all conditions; no recommendation can take into account all of the unique features of individual patients and clinical circumstances.

improve quality of life and stop disease progression. Cough and sputum production, along with breathlessness are the most frequent symptoms but rhinosinusitis, fatigue, haemoptysis and thoracic pain are also common [4]. Quality of life impairment in bronchiectasis is equivalent in terms of scores on the St George's Respiratory Questionnaire (SGRQ) to severe chronic obstructive pulmonary disease (COPD), idiopathic pulmonary fibrosis and other disabling respiratory diseases $[5,6]$.

Exacerbations of bronchiectasis are key targets for therapy as they are major determinants of healthcare costs. They are associated with increased airways and systemic inflammation [7] and progressive lung damage $[8,9]$. In addition, more severe and more frequent exacerbations are associated with worse quality of life, daily symptoms [10], lung function decline [11], and mortality [9]. Consequently, the majority of therapeutic interventions are aimed at reducing exacerbations. Despite current treatment approaches, European registry data shows that approximately $50 \%$ of European bronchiectasis patients have two or more exacerbations per year and one third require at least one hospitalisation per year [12].

Our understanding of what causes symptoms and exacerbations is based on the vicious cycle concept, with key components of the disease being chronic bronchial infection, inflammation, impaired mucociliary clearance and structural lung damage. Treatment is primarily based on the principles of preventing or suppressing acute and chronic bronchial infection, improving mucociliary clearance and reducing the impact of structural lung disease (figure 1).

Chronic airways infection, most frequently with Haemophilus influenzae and Pseudomonas aeruginosa and less frequently with Moraxella catarrhalis, Staphylococcus aureus and Enterobacteriaceae, stimulate and sustain lung inflammation. Persistent isolation of these organisms in sputum or bronchoalveolar lavage is associated with an increased frequency of exacerbations, worse quality of life and increased mortality $[13,14]$. This is particularly the case with $P$. aeruginosa infection. A systematic review of observational studies identified that $P$. aeruginosa infection is associated with a three-fold increase in mortality risk, an almost seven-fold increase in risk of hospital admission and an average of one additional exacerbation per patient per year [15].

Inflammation in bronchiectasis is primarily neutrophilic and closely linked to persistent bacterial infection. Excessive neutrophilic inflammation is linked to an increased frequency of exacerbations and rapid lung function decline through degradation of airway elastin, among other mechanisms [16-19]. The available data also support a role for cell-mediated immunity, specifically T-cells in the pathophysiology of bronchiectasis, but the role of other inflammatory cells is less clear [17].

Mucociliary clearance is impaired by the impact of structural bronchiectasis, airway dehydration, excess mucus volume and viscosity. More than $70 \%$ of bronchiectasis patients expectorate sputum daily with highly variable sputum volumes. Treatment aims to prevent mucus stasis and the associated mucus plugging, airflow obstruction and progressive lung damage [20].

Structural changes in the lung associated with disease include bronchial dilatation, bronchial wall thickening, and mucus plugging as well as small airways disease and emphysema. More than 50\% of patients have airflow obstruction, but restrictive, mixed ventilatory pattern and preserved lung function are 


\section{Chronic bronchial infection}

Long-term inhaled or oral antibiotic therapy

Eradication of new pathogenic microorganisms

Antibiotic treatment of exacerbations

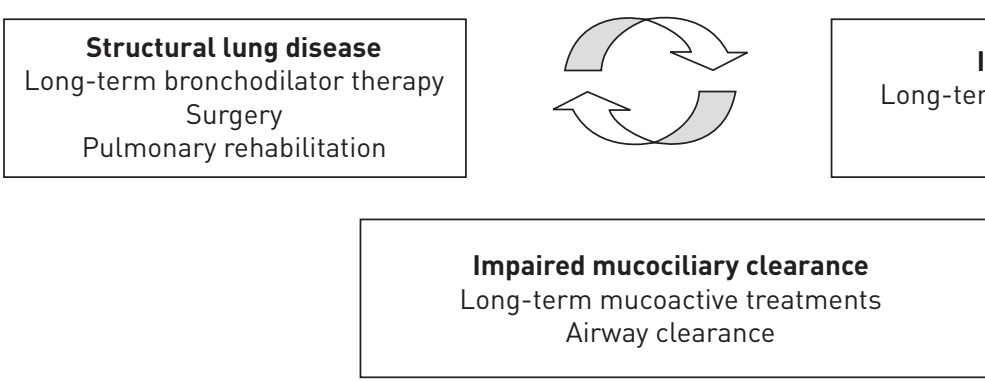

FIGURE 1 Treatments for bronchiectasis considered in this guideline according to the vicious cycle concept of bronchiectasis.

also frequently observed. Breathlessness is due to the impact of airflow obstruction, impaired gas transfer, exercise deconditioning and the impact of comorbidities [21-24]. Breathlessness is one of the strongest predictors of mortality $[9,14]$. Therapies may aim to treat airflow obstruction (e.g. bronchodilators), to improve exercise capacity (pulmonary rehabilitation), or to remove poorly functioning or diseased lung (e.g. surgery).

Bronchiectasis has long been a neglected disease. The prevalence of bronchiectasis has been estimated at 53 to 566 cases per 100000 inhabitants. Prevalence increases with age and female gender [25-29].

QuiNT et al. [28] described that age-adjusted mortality rate for bronchiectasis was 1437.7 per 100000 . Several longitudinal studies have described up to a $30 \%$ mortality at 1-year follow-up after suffering an exacerbation [30,31], particularly in the presence of COPD [32].

The economic burden of this disease has been estimated to be similar to COPD; this increases with disease severity, hospitalisations, need for intensive care, and use of inhaled antibiotics $[25,26,30,33,34]$. No therapies are currently specifically licensed by regulatory authorities in Europe or the USA for the treatment of bronchiectasis. Historically, treatment has been extrapolated from the management of cystic fibrosis bronchiectasis, but randomised clinical trials and clinical experience has demonstrated that treatment responses are different and that specific guidance for bronchiectasis not due to cystic fibrosis is necessary $[35,36]$.

National guidelines are available in Europe: the Spanish guidelines (SEPAR) were published in 2008 [37] and the British Thoracic Society (BTS) guidelines were published in 2010 [38]. Aspects of management of acute exacerbations in bronchiectasis were addressed in the European Respiratory Society (ERS)/European Society for Clinical Microbiology and Infectious Diseases lower respiratory tract infections guidelines published in 2011 [39]. However, to date, there are no international guidelines for the management of adult bronchiectasis published and no national guidelines published in Europe in the past 5 years.

\section{Methods}

This guideline was developed by a European Respiratory Society bronchiectasis task force chaired by E. Polverino (Spain) and J.D. Chalmers (UK). The task force included specialists in respiratory medicine with recognised expertise in the management of patients with lung infections, as well as a microbiologist, an immunologist, a physiotherapist, a general practitioner, a thoracic surgeon, three patient representatives from the European Multicentre Bronchiectasis Audit and Research Collaboration (EMBARC)/European Lung Foundation (ELF) bronchiectasis patient advisory group and two ERS methodologists.

The guideline panel held four face-to-face meetings, beginning in January 2015. The most relevant clinical questions on the management of bronchiectasis in adults (for both clinicians and patients) were debated. A total of nine clinical questions were formulated using the PICO format (Patients, Intervention, Comparison, Outcomes) and systematic reviews were conducted to answer these specific questions, until September 2016 when the final guideline recommendations were discussed and agreed. Regular teleconferences and discussions via e-mail around individual topics were held. The patient representatives were actively involved in all discussions as full members of the guideline committee, provided input into the final recommendations and will be involved in developing a lay version of the guideline. 


\section{Disclosure of potential conflicts of interest}

Committee members disclosed all potential conflicts of interest according to ERS policy. Conflicted members were asked to abstain from discussions and voting on recommendations in which they were considered to have potential conflicts. Compliance with the conflict of interest policy was monitored by the chairs. The methodologists were non-voting members of the panel.

\section{Systematic review}

An experienced external librarian designed and ran a search strategy using MeSH terms and keywords for each clinical question, in collaboration with the methodologists. More details of the search strategy are shown in the supplementary material. The search retrieved 3038 records; after removal of duplicates and exclusion of citations that did not meet the established inclusion criteria, a total of 48 references were included in the evidence summaries (figure 2; supplementary material).

\section{Assessment of the level of evidence and degree of recommendations}

The panel selected outcomes of interest for each clinical question a priori, based on their relative importance to adult patients with bronchiectasis and to clinical decision making (supplementary material).

We followed the GRADE approach to assess the confidence in the evidence (quality) and the degree of recommendations [2]. Recommendations are graded as strong or conditional after considering the quality of the evidence, the balance of desirable and undesirable consequences of compared management options, the assumptions about the relative importance of outcomes, the implications for resource use, and the acceptability and feasibility of implementation [40].

Evidence summary of findings tables and evidence to decisions frameworks were generated for each clinical question (supplementary material) [41]. Based on these formats, the panel formulated the clinical recommendations and decided on their strength by consensus, or, if required, by voting. Following the GRADE approach, strong recommendations are worded as "we recommend", while conditional recommendations are worded as "we suggest".

\section{Question 1: Is standardised testing for the cause of bronchiectasis beneficial when compared with no standardised testing? \\ Recommendations}

We suggest the minimum bundle of aetiological tests in adults with a new diagnosis of bronchiectasis (conditional recommendation, very low quality of evidence) is: 1) differential blood count; 2) serum immunoglobulins (total IgG, IgA and IgM); and 3) testing for allergic bronchopulmonary aspergillosis (ABPA).

It is expected that sputum culture is undertaken for monitoring purposes of bacterial infection. Mycobacterial culture may be helpful in selected cases where NTM are suspected as an aetiological cause of bronchiectasis. Additional tests may be appropriate in response to specific clinical features, or in patients with severe or rapidly progressive disease.

\section{Summary of the evidence}

The SEPAR and BTS guidelines have previously recommended a routine "bundle" of tests at diagnosis to identify possible underlying causes of bronchiectasis [37, 38]. Our systematic review identified no publications which directly addressed whether routine aetiological investigation protocols provide benefit compared to clinically driven investigations or no testing. Four observational studies were identified which describe the percentage of adult patients (7-37\%) whose management changed following investigation of aetiology while no other relevant outcomes were reported [42-45].

\section{Justification of the recommendation}

Measurement of circulating white cell count and differential is suggested in all patients. The presence of lymphopenia or neutropenia may suggest primary or secondary immune deficiency, while lymphocytosis may suggest secondary immune deficiency as a consequence of haematological malignancy.

Serum IgA, IgM, IgG are generally tested together, and we have considered them jointly. Low IgG, with or without low IgM or low IgA may indicate a defective antibody production that is an important modifiable cause of bronchiectasis, and $2-8 \%$ of patients with bronchiectasis have common variable immune deficiency [42-44]. Importantly, in these cases immunoglobulin replacement treatment can result in significant improvement in short and long-term outcomes. The cost of serum immunoglobulin testing is low and testing is readily available. 




FIGURE 2 PRISMA flow diagram.

The geographic distribution of ABPA is thought to be variable, but establishing diagnosis alters management [45]. Hence the panel advises routine screening of all patients for ABPA at diagnosis. The generally recommended screening tests for ABPA are total serum IgE, specific IgG to Aspergillus, and specific IgE to Aspergillus or, as an alternative, skin prick tests to Aspergillus [46, 47].

A range of other tests may be appropriate in specific circumstances. In patients with radiological features of NTM or clinical features such as weight loss, haemoptysis, rapid deterioration or symptoms non-responsive to standard therapy, three sequential daily sputum cultures for mycobacterial cultures or a single bronchoalveolar lavage should be considered [48]. Some authorities recommend measuring antibody responses to S. pneumoniae 23 valent polysaccharide vaccine (PPV23) in order to identify individuals with specific polysaccharide antibody deficiency $[37,38]$. Failure to make an antibody response to PPV23 (four-fold increase in titre at 4-6 weeks) may suggest a defect in carbohydrate antigen responses. However, due to the large variability in individual antibody response to PPV23 and in testing protocols, this 
evaluation should not be performed without specialist support. Testing for cystic fibrosis with measurement of sweat chloride, other biomarkers of cystic fibrosis transmembrane conductance regulator (CFTR)-mediated chloride ion transport and CFTR gene mutation analysis should be considered in young adults or with specific clinical features of cystic fibrosis, such as upper lobe predominance of bronchiectasis on chest $\mathrm{CT}$, the presence of nasal polyposis and/or chronic rhinosinusitis, recurrent pancreatitis, male primary infertility and/or malabsorption. Testing for primary ciliary dyskinesia with nasal nitric oxide, high-speed video analysis, transmission electron microscopy, immunofluorescence and/ or genetic testing should be considered for patients with several of the following features: persistent wet cough since childhood, situs anomalies, congenital cardiac defects, nasal polyposis and/or chronic rhinosinusitis, chronic middle ear disease with or without hearing loss, a history of neonatal respiratory distress or neonatal intensive care admittance in term infants. Refer to the ERS guidelines for the diagnosis of primary ciliary dyskinesia for more information [49]. The presence of basal emphysema or early onset airflow obstruction could suggest the need to exclude alpha1-antitrypsin deficiency. There are a wide range of other causes of bronchiectasis many of which can be identified by history, physical examination and CT scanning. We do not recommend routine testing of autoantibodies to screen for connective tissue disease, but evidence of connective tissue disease should be sought by history and physical examination.

The suggested bundle is justified by the fact that, despite the lack of strong evidence, selected tests can considerably alter the clinical management of bronchiectasis by indicating specific therapeutic interventions such as immunoglobulin replacement, corticosteroids or antifungal treatment. These interventions imply significant potential benefits for some individuals, and minimal undesirable effects from testing for others. The patient advisory group reported that patients placed a high value on identifying the underlying cause of bronchiectasis.

\section{Implementation considerations}

The standard tests recommended in this bundle should be available in the majority of healthcare systems and should not present major implementation issues.

\section{Question 2: Are courses of 14-21 days of systemic antibiotic therapy compared to shorter courses (<14 days) beneficial for treating adult bronchiectasis patients with an acute exacerbation? \\ Recommendation}

We suggest acute exacerbations of bronchiectasis should be treated with 14 days of antibiotics (conditional recommendation, very low quality of evidence).

\section{Summary of the evidence}

Bronchiectasis patients are typically given prolonged courses of antibiotics of 14 days' duration for infective exacerbations. This recommendation is given in previous guidelines for bronchiectasis [37, 38]. It is based on expert consensus and studies that documented good clinical outcomes with such treatment regimens. However, the evidence base for this duration is poor.

The published literature was assessed as to whether shorter ( $<14$ days) courses of antibiotics would be as clinically effective or be associated with any harm compared to 14-21 days of therapy. There was no direct evidence of benefit favouring either 14-21 days or shorter courses of antibiotic therapy. The only data comes from an indirect comparison of response at day 7 versus day 14 in 53 patients, all receiving ciprofloxacin (with or without inhaled tobramycin) for 14 days. After pooling both study arms, bacterial load (MD: $+0.23 \mathrm{cfu} \cdot \mathrm{mL}^{-1}$ higher at day $14,95 \% \mathrm{CI}-1.55$ to +2.01$)$ and forced expiratory volume in $1 \mathrm{~s}$ (FEV1) (MD: $+0.01 \mathrm{~L}$ at day $14,95 \% \mathrm{CI}-0.51$ to +0.53 ) were similar at 7 and 14 days with wide confidence intervals including both benefit and harm. No data was available for clinical outcome such as subsequent quality of life and exacerbations (supplementary material) [50].

\section{Data from other studies}

Some authors have shown a favourable impact of 14 days of antibiotics for treatment of a bronchiectasis exacerbation. One study of 32 exacerbations treated with 14 days of intravenous antibiotics demonstrated significant improvement in 24-h sputum volume, bacterial clearance, C-reactive protein, incremental walk test and SGRQ, but no improvement in spirometry [51]. A further study of 34 patients treated with intravenous antibiotics for 14 days demonstrated a reduction in sputum bacterial load and markers of airway inflammation after antibiotic treatment [7].

\section{Justification of the recommendations}

In the absence of any direct data comparing longer and shorter courses of antibiotics, we suggest continuing the usual practice of treating acute exacerbations of bronchiectasis with 14 days of antibiotics 
on the basis of the patient's prior microbiology testing and the severity of the exacerbation. Patients have diverse views on the duration of antibiotics for exacerbations, with some preferring longer courses, and other patients wishing to use shorter courses if possible.

\section{Implementation considerations}

It is possible that shorter courses of antibiotics may be appropriate in some cases. The task force panel suggests that mild exacerbations, exacerbations in mild patients, those associated with pathogens more sensitive to antibiotics (e.g. S. pneumoniae), or patients with a rapid return to baseline state may benefit from shorter courses, but evidence supporting shorter course treatment is lacking. Otherwise, in patients with lack of recovery by 14 days of antibiotic therapy we suggest re-evaluation of the patient's clinical condition and a new microbiological investigation. Sending a sputum sample at the start of an exacerbation is helpful to guide choice of antibiotics in the event of inadequate response to initial therapy. Due to variations in antibiotic use and healthcare practices across Europe, we do not address choice of specific antibiotics, or the role of combination versus monotherapy in this guideline.

Further research studies assessing the optimal duration of antibiotics are recommended.

Question 3: Is eradication treatment beneficial for treating bronchiectasis patients with a new isolate of a potentially pathogenic microorganism in comparison to no eradication treatment?

Recommendations

We suggest that adults with bronchiectasis with a new isolation of $P$. aeruginosa should be offered eradication antibiotic treatment (conditional recommendation, very low quality of evidence).

We suggest not offering eradication antibiotic treatment to adults with bronchiectasis following new isolation of pathogens other than $P$. aeruginosa (conditional recommendation, very low quality of evidence)

\section{Summary of the evidence}

Eradication treatment refers to any antibiotic treatment given with the express intention of achieving complete clearance of the pathogen from the airway. In bronchiectasis, eradication treatment regimens vary, but there is some evidence suggesting that a regimen including a nebulised antibiotic achieves greater rates of clearance and clinical benefits than intravenous treatment alone in achieving clearance of $P$. aeruginosa [52].

Chronic airway infection in adult patients with bronchiectasis is frequent and usually associated with worse outcomes such as more exacerbations and poorer quality of life [15, 53]. Definitions of chronic airway infection in bronchiectasis are not established but a systematic review identified that the most frequent definition used in bronchiectasis studies is two or more isolates of the same organism at least 3 months apart in 1 year [15]. Unfortunately, in patients with persistent infections, there is little evidence about the beneficial effects of pathogen eradication beyond $P$. aeruginosa.

We could not identify any randomised controlled trial directly addressing the question. Therefore we included two studies that investigated whether eradication treatment in adult patients with bronchiectasis improved clinical outcomes compared to the patient's own baseline [54, 55]. Pooled analysis provides some evidence of the potential benefits of $P$. aeruginosa eradication in terms of negative sputum samples, frequency of subsequent exacerbations and quality of life, but the evidence is indirect and considered of low quality.

In particular, the retrospective observational study of WHITE et al. [55] analysed different eradication treatment regimens: i.v. antibiotics (12 cases), i.v. antibiotics followed by inhaled antibiotics (13 cases), and oral ciprofloxacin alone. 25 patients across all groups received 3 months of inhaled colistin. Initial clearance rate from sputum was $80 \%$, but $54 \%$ of all patients remained $P$. aeruginosa free at follow-up and the exacerbation rate fell from 3.93 to 2.09 per year after the eradication treatment. In addition, two thirds of patients experienced clinical improvement although lung function remained unchanged.

ORRIOLS et al. [54] performed a 15-month single-masked, randomised controlled trial in 35 patients with early $P$. aeruginosa infection. These patients received initial therapy with i.v. ceftazidime or tobramycin followed by 3 months of $300 \mathrm{mg}$ of nebulised tobramycin b.d. or placebo. At the end of follow-up (12 months), 54\% of patients were free of P. aeruginosa in the tobramycin group versus $29 \%$ in the placebo group. Despite some potential methodological limitations, this study showed that the median time to recurrence of $P$. aeruginosa was longer in the treatment arm compared to placebo and numbers of exacerbations and hospital admissions were lower in the nebulised tobramycin group. The impact of eradication treatments on the development of antibiotic resistance was not extensively studied. This study had no control group and so is limited in terms of informing whether eradication is effective. 


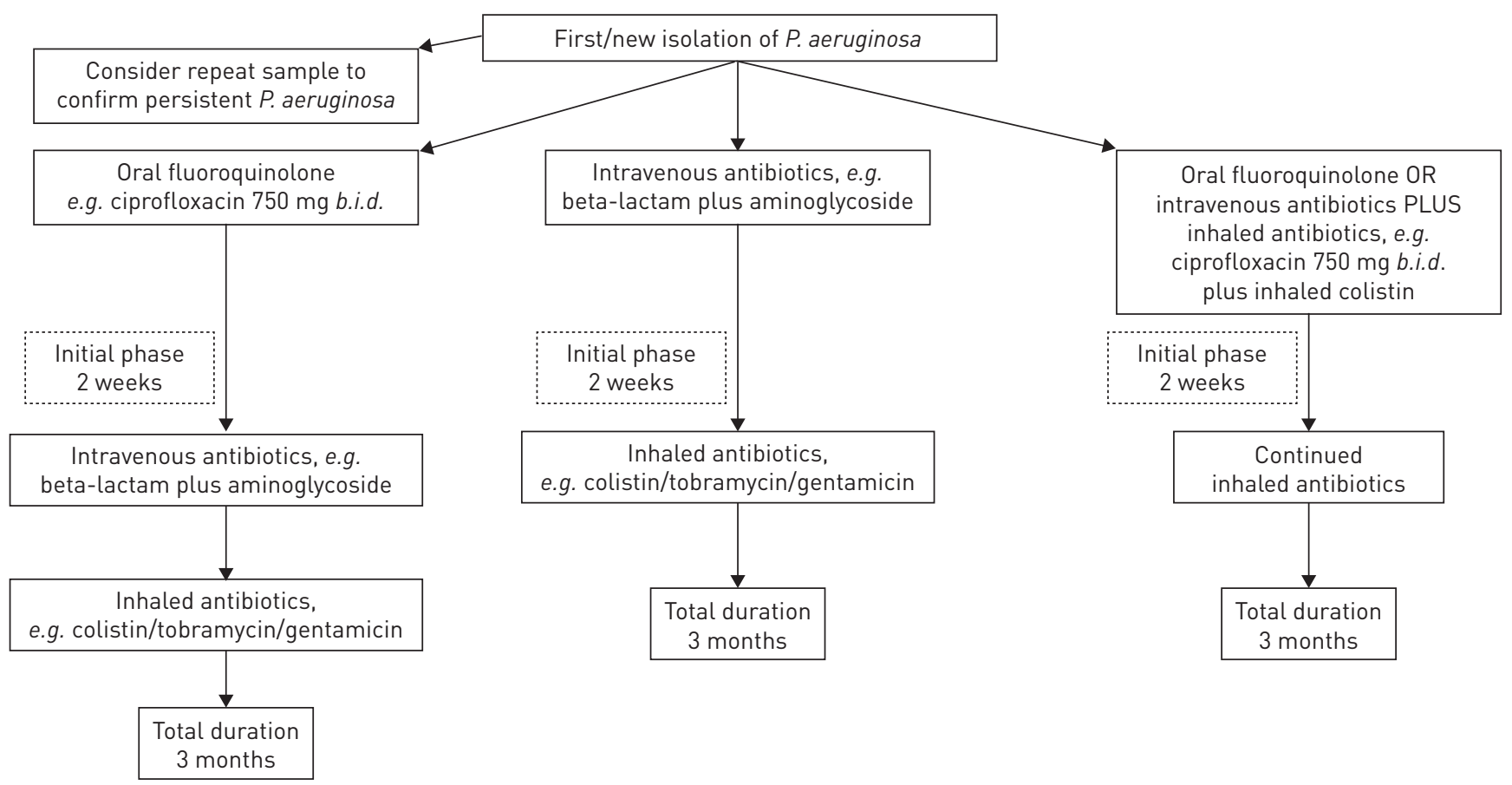

FIGURE 3 Three possible and alternative eradication treatment pathways based on what is commonly used in clinical practice. After each step it is recommended to repeat sputum sampling for Pseudomonas aeruginosa and to progress to the next step if the culture remains positive.

There is no clear evidence to support one regimen over another, and therefore figure 3 illustrates some commonly used regimes.

\section{Justification of the recommendation}

The poor clinical outcomes associated with chronic $P$. aeruginosa infection, the data from one observational study and the clinical experience in cystic fibrosis suggests that $P$. aeruginosa eradication may positively influence important clinical outcomes including exacerbation frequency.

There is no evidence to support eradication of organisms other than P. aeruginosa and in organisms that are not so clearly associated with poorer outcomes, the risk-benefit ratio is less in favour of eradication treatment.

\section{Implementation considerations}

Identification of new isolates of $P$. aeruginosa requires regular sputum surveillance which has resource implications. We suggest as a minimum that patients should have a sputum sample sent when clinically stable once per year. In circumstances where the date of acquisition of $P$. aeruginosa is uncertain, a clinical judgement must be made on the likely success or otherwise of an eradication attempt. This guideline does not address attempted eradication of chronic P. aeruginosa infection, where the infection has been present for many years, as this is thought unlikely to be successful. The quality of evidence is low and further research is also needed on potential side effects of eradication therapies and, particularly, the emergence of resistance or new infections.

\section{Question 4: Should long-term anti-inflammatory agents be used in adult patients with bronchiectasis? \\ Recommendation:}

We suggest not offering treatment with inhaled corticosteroids to adults with bronchiectasis (conditional recommendation, low quality of evidence).

We recommend not offering statins for the treatment of bronchiectasis (strong recommendation, low quality of evidence).

We suggest that the diagnosis of bronchiectasis should not affect the use of inhaled corticosteroids in patients with comorbid asthma or COPD (best practice advice, indirect evidence). 
We considered only studies of anti-inflammatory drugs that were at least 3 months in duration. Although macrolides may have anti-inflammatory activity their role in bronchiectasis is discussed within PICO question 5 of these guidelines (regarding antibiotics). We identified six systematic reviews [56-61] and three studies that met our inclusion criteria [62-64].

HERNANDo et al. [64] reported a double-blind randomised controlled trial over 6 months with 77 patients allocated to inhaled budesonide $400 \mu \mathrm{g}$ b.d. or placebo with a primary outcome of lung function. Tsang et al. [62] reported a trial of inhaled fluticasone versus placebo over 12 months in 86 patients with co-primary end-points of $24 \mathrm{~h}$ sputum volume and annual exacerbation frequency. MANDAL et al. [63] studied atorvastatin in 30 patients over 6 months compared to a matched group receiving placebo with a primary outcome improvement in cough related quality of life measured by the Leicester cough questionnaire. Overall the three studies included only 193 patients. Two of the studies assessed the effects of anti-inflammatories on exacerbations with a wide confidence interval (rate ratio (RR) 0.99, 95\% CI 0.76-1.30) $[62,64]$. Hence no clear benefit on reducing exacerbations was noted.

The effect on quality of life, using the SGRQ, was only reported in two studies (123 patients) [63, 64], with an observed improvement of 0.91 points (below the minimal clinically significant difference of 4 points, $95 \% \mathrm{CI}-4.51$ to +6.33 ). All three studies reported FEV1 and forced vital capacity (FVC) as lung function outcomes [62-64]. No significant benefit was seen with any of the treatments studied for lung function.

The study design and small number of patients make these studies not optimal for safety assessment. Across the three studies the pooled estimate of suffering any adverse event was RR 2.75 (95\% CI 1.21-6.25) as compared to control. The adverse effect profile of both inhaled corticosteroids and statins has been well described.

The increase in pooled adverse events was largely driven by MANDAL et al. [63] who reported that adverse events led to withdrawal from the atorvastatin group (one case of headache, one of diarrhoea, and two of combined diarrhoea and headache). One was withdrawn due to liver function abnormalities at 3 months. $10(33 \%)$ patients receiving atorvastatin had an adverse event versus three (10\%) allocated placebo (difference 23\%, 95\% CI 3-43; $\mathrm{p}=0.02$ ).

For the inhaled corticosteroid trials in bronchiectasis, adverse event reporting was incomplete. Known and frequent local adverse events across all diseases include dysphonia and oropharyngeal candidiasis. More severe adverse events include: alteration of the hypothalamic-pituitary-adrenal axis function, pneumonia, increased intraocular pressure, formation of cataracts and decreased bone density.

\section{Justification of recommendations}

There are no large trials of anti-inflammatory therapies in bronchiectasis and the existing studies show minimal and, in most cases, no clinically significant benefits. The increased frequency of adverse events, particularly with statins, justifies a recommendation against their use. The guideline panel concludes that inhaled corticosteroids do not have a role in the routine management of bronchiectasis. Inhaled corticosteroids have an established role in the treatment of asthma and a proportion of patients with COPD. In the absence of specific data in adult patients with bronchiectasis and these two conditions, the guideline panel concludes that the presence of bronchiectasis alone should not lead to a decision to withdraw inhaled corticosteroids from patients with established asthma or COPD.

\section{Implementation considerations}

We recommend randomised controlled trials of inhaled corticosteroids in bronchiectasis who are naïve to inhaled corticosteroid therapy. Inhaled corticosteroid use is, however, already widespread in bronchiectasis. In those already treated with inhaled corticosteroids and no clear history of asthma or COPD a randomised controlled trial of inhaled corticosteroid withdrawal may help define true utility of this widely prescribed therapy.

\section{Question 5: Is long-term antibiotic treatment ( $\geqslant 3$ months) compared to no treatment beneficial for treating adult bronchiectasis patients? \\ Recommendations}

We suggest offering long-term antibiotic treatment for adults with bronchiectasis who have three or more exacerbations per year (conditional recommendation, moderate quality evidence).

All subsequent recommendations refer to patients with three or more exacerbations per year.

We suggest long-term treatment with an inhaled antibiotic for adults with bronchiectasis and chronic $P$. aeruginosa infection (conditional recommendation, moderate quality evidence). 
We suggest long-term treatment with macrolides (azithromycin, erythromycin) for adults with bronchiectasis and chronic $P$. aeruginosa infection in whom an inhaled antibiotic is contraindicated, not tolerated or not feasible (conditional recommendation, low quality evidence).

We suggest long-term treatment with macrolides (azithromycin, erythromycin) in addition to or in place of an inhaled antibiotic, for adults with bronchiectasis and chronic P. aeruginosa infection who have a high exacerbation frequency despite taking an inhaled antibiotic (conditional recommendation, low quality evidence).

We suggest long-term treatment with macrolides (azithromycin, erythromycin) for adults with bronchiectasis not infected with $P$. aeruginosa (conditional recommendation, moderate quality evidence).

We suggest long-term treatment with an oral antibiotic (choice based on antibiotic susceptibility and patient tolerance) for adults with bronchiectasis not infected with $P$. aeruginosa in whom macrolides are contraindicated, not tolerated or ineffective (conditional recommendation, low quality evidence).

We suggest long-term treatment with an inhaled antibiotic for adults with bronchiectasis not infected with $P$. aeruginosa in whom oral antibiotic prophylaxis is contraindicated, not tolerated or ineffective (conditional recommendation, low quality of evidence).

We identified eight systematic reviews [65-72] and 17 relevant studies for this clinical question [36, 52, 73-86]. Our evidence summary suggests that long-term antibiotic use, pooling both inhaled and oral antibiotic data, reduces the number of exacerbations, time to first exacerbation, sputum purulence and breathlessness in adults with bronchiectasis; however, they are also associated with more adverse events and bacterial resistance.

Three randomised controlled trials evaluating the effect of nebulised antibiotics in adults with bronchiectasis suggested beneficial effects on exacerbation frequency and/or time to first exacerbation [65, $76,78,83$ ]. In a study involving 144 adults with bronchiectasis and P. aeruginosa infection, colistin $1 \mathrm{MU}$ delivered twice daily through the I-neb was not associated with a statistically significant improvement in time to first exacerbation compared to placebo [83]. However, in a pre-planned analysis in adherent individuals (defined as taking $\geqslant 81 \%$ of doses recorded by the I-neb), the median (25th quartile) time to exacerbation was 168 (65) days versus 103 (37) days in the colistin and placebo groups, respectively $(\mathrm{p}=0.038)$. A similar treatment effect was reported in a study evaluating nebulised liposomal ciprofloxacin in 42 adult patients with bronchiectasis and P. aeruginosa infection [78]. A 12-month single blind study of nebulised gentamicin in 65 adults with bronchiectasis predominantly infected with $H$. influenzae ( $\mathrm{n}=26$, $46 \%$ ) or $P$. aeruginosa $(\mathrm{n}=24,42 \%)$ showed significant benefits including fewer exacerbations compared to $0.9 \%$ saline-treated patients [76].

Three randomised controlled trials showed beneficial effects of macrolide antibiotics (azithromycin or erythromycin) on exacerbation frequency in adults with bronchiectasis: EMBRACE (141 patients on azithromycin or placebo for 6 months) [77], BAT ( 83 patients on azithromycin or placebo for 12 months) [80] and BLESS (117 patients on erythromycin or placebo for 12 months) [79]. The EMBRACE study showed the rate of event-based exacerbations was 0.59 per patient in the azithromycin group and 1.57 per patient in the placebo group in the 6-month treatment period (RR $0.38,95 \%$ CI $0.26-0.54 ; \mathrm{p}<0.0001$ ); the BAT study showed the median (interquartile range) number of exacerbations in the azithromycin group was $0(0-1)$, compared with $2(1-3)$ in the placebo group $(\mathrm{p}<0.001)$; and the BLESS study showed erythromycin significantly reduced protocol defined exacerbations compared to placebo (mean 1.29, 95\% CI $0.93-1.65$ versus $1.97,95 \%$ CI $1.45-2.48$ per patient per year; $\mathrm{p}<0.003$ ). Doses used in clinical trials or in clinical practice range from $250 \mathrm{mg}$ azithromycin daily, $500 \mathrm{mg}$ or $250 \mathrm{mg}$ three times per week, and erythromycin $400 \mathrm{mg}$ twice daily.

Historical randomised controlled trials evaluating penicillin and tetracycline based antibiotic regimens also suggest some benefit in adults with bronchiectasis, with two long-term studies reporting less days off work and reduced sputum purulence with oxytetracycline [84] or amoxicillin treatment [73].

Important adverse events were reported with long-term antibiotic treatment. Diarrhoea was more common with oral antibiotics than placebo in the macrolide studies, although treatment discontinuation was rare [66-70]. There was also a $28 \%$ increase in the proportion of macrolide-resistant commensal oropharyngeal Streptococci after 12 months treatment with erythromycin and a macrolide resistance rate of $88 \%$ following 12 months of azithromycin [79, 80]. In contrast, there was no antimicrobial resistance reported after 612 months of nebulised colistin, dual release liposomal ciprofloxacin or gentamicin $[76,78,83]$. While these specific nebulised preparations were well tolerated, two phase III trials reported more frequent treatment-related adverse events (1.4 and 1.8 times greater) and discontinuations (2.1 and 6.7 times greater) associated with nebulised aztreonam compared to placebo [36]. The most commonly reported 
adverse events were breathlessness, cough and increased sputum production. The incidence of potential treatment-related adverse events such as QTc prolongation with macrolides, tinnitus/hearing loss with macrolides and inhaled aminoglycosides, and renal dysfunction with inhaled aminoglycosides is not known in people with bronchiectasis, but should be considered when weighing up the potential benefits and harms of long-term antibiotic treatment.

\section{Justification of recommendations}

The overall balance of desirable effects (particularly fewer exacerbations), undesirable effects (particularly gastrointestinal upset and antimicrobial resistance) and patient values favours long-term antibiotic treatment in selected patients (figure 4). For individuals with $P$. aeruginosa, the currently available evidence supports continuous use of nebulised colistin [83] or gentamicin [76]). Nebulised aztreonam is not recommended due to the lack of efficacy with regard to quality of life improvement over two treatment cycles and a high adverse event rate reported in the pivotal phase III trials [36]. Due to the relatively low number of participants with $P$. aeruginosa in the macrolide studies, the use of macrolide antibiotics is suggested as a second-line option in patients with this organism $[77,79,80]$. However, for individuals with no evidence of $P$. aeruginosa infection, macrolide treatment is suggested as first-line treatment due to the high-quality evidence for exacerbation reduction and an acceptable side-effect profile $[77,79,80]$.

Although the macrolide studies included a minimum exacerbation frequency of one [77], two [79] or three [80] exacerbations in the year preceding enrolment as an entry criterion, the mean exacerbation frequency in the year prior to enrolment in each of the three studies was $\geqslant 3$. Due to potential undesirable effects, the suggested threshold for starting long-term antibiotic treatment is $\geqslant 3$ exacerbations per year. However, this threshold may be reduced for individuals with: a history of severe exacerbation, relevant comorbidities such as primary/secondary immunodeficiency, patients in whom exacerbations are having a significant impact on their quality of life or those with more severe bronchiectasis [9].

Before considering the prescription of long-term antibiotics, general aspects of bronchiectasis management need to be optimised, such as airway clearance and treating modifiable underlying causes. Careful characterisation of sputum pathogens (bacteria, mycobacteria and fungi) before and after implementation of long-term antibiotics is essential to direct antibiotic choices, monitor resistance patterns and identify treatment emergent organisms. Drug toxicity monitoring is also required, most notably with macrolides and inhaled aminoglycosides.

\section{Implementation considerations}

The use of inhaled antibiotics is associated with a 10-32\% risk of bronchospasm [76, 87] and a supervised test dose with pre- and post-spirometry is recommended. Prior inhalation of a short-acting bronchodilator may prevent bronchospasm and, therefore, is advisable.

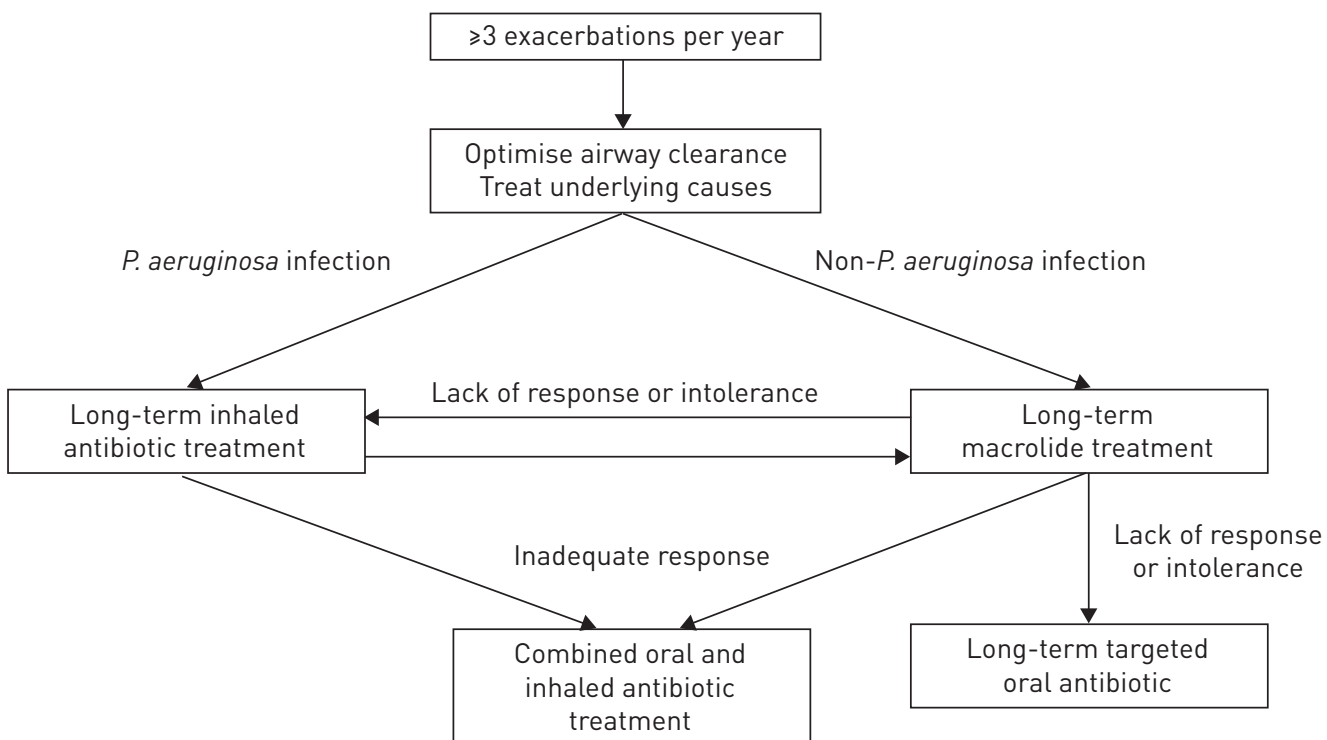

FIGURE 4 Summary of recommendations for long-term antibiotic treatment. 
Prior to long-term treatment with macrolides, we recommend excluding active NTM infection because macrolide monotherapy can increase the risk of macrolide resistance in NTM.

No cost-effectiveness studies were identified regarding the use of long-term antibiotics in adult patients with bronchiectasis and further research will be required to determine if cyclical or continuous treatment (possibly involving combinations of preparations) is optimal in terms of exacerbation frequency reduction, treatment burden and risk of antimicrobial resistance.

Figure 4 summarises the approach to long-term antibiotic treatment in adults with bronchiectasis summarising the above guideline recommendations.

\section{Question 6: Is long-term mucoactive treatment ( $\geqslant 3$ months) compared to no treatment beneficial for treating adult bronchiectasis patients? Recommendation}

We suggest offering long-term mucoactive treatment ( $\geqslant 3$ months) in adult patients with bronchiectasis who have difficulty in expectorating sputum and poor quality of life and where standard airway clearance techniques have failed to control symptoms (weak recommendation, low quality evidence).

We recommend not to offer recombinant human DNase to adult patients with bronchiectasis (strong recommendation, moderate quality evidence)

\section{Summary of the evidence}

Airway clearance adjuncts such as mucolytics and hyperosmolar agents alter mucus viscosity and/or enhance mucociliary clearance. We identified three systematic reviews [88-90] and five relevant studies meeting inclusion criteria for this clinical question [35, 91-94]. Of these five randomised controlled trials, two were performed with dry powder mannitol at doses of $320 \mathrm{mg}(\mathrm{n}=343)$ [93] and $400 \mathrm{mg}$ twice daily $(\mathrm{n}=461)$ [94], one with nebulised recombinant human DNase at a dose of $2.5 \mathrm{mg}$ twice daily $(\mathrm{n}=349)$ [35], and two with nebulised hypertonic saline (one with $4 \mathrm{~mL} \mathrm{7 \%}$ once daily [91] or $5 \mathrm{~mL} 6 \%$ twice daily [92], $\mathrm{n}=28$ and $\mathrm{n}=40$ respectively). Only in two studies $[35,93]$ was the treatment compared to placebo. In one study with mannitol [94] and in both studies with hypertonic saline [91, 92], the treatment was compared to low dose mannitol (50 mg twice daily) [94] and isotonic saline [91], respectively. Three previous meta-analyses of mucoactive and inhaled hyperosmolar agents in bronchiectasis prior to the most recent mannitol study [93] found insufficient evidence to draw firm conclusions on the effect of inhaled mucoactive and hyperosmolar treatment due to the significant differences in methodology, patient groups and findings amongst the limited data available [88-90].

Patients with $\geqslant 2$ exacerbations in the previous year and a baseline minimum SGRQ score of 30 who received mannitol showed a significantly greater improvement in total SGRQ score compared to controls (low dose mannitol), although the difference between arms did not reach the minimal clinically important difference for the total SGRQ score [93]. An improvement in SGRQ components was shown in patients without chronic $P$. aeruginosa infection and with no long-term antibiotic treatment who received hypertonic saline 7\% [92].

None of the mucoactive agents significantly reduced the number of exacerbations, and the exacerbation rate was higher in the rhDNase group compared with placebo [35]. In patients with $\geqslant 2$ exacerbations in the previous year, mannitol increased the time to first exacerbation [94]. In one study with hypertonic saline $7 \%$ there were reductions in health care utilisation when comparing prospectively collected data between hypertonic saline and isotonic saline phases [92].

In four studies, a tolerance test was performed at first administration: patients with mannitol-induced bronchospasm (16\%) [93, 94] or a decrease in FEV1 of more than 10\% [92] or 15\% [91] after inhalation of hypertonic saline ( $7 \%$ and $6 \%$, respectively) were excluded.

In the 3-month study, $1.8 \%$ of patients randomised to mannitol experienced bronchospasm and $1.3 \%$ reported dyspnoea as opposed to none in the placebo group [93]. In the 13-month study, $20.2 \%$ of patients in the mannitol arm and $16.7 \%$ in the control group experienced adverse events related to study medication, most of which were judged to be mild or moderate [94]. Hypertonic saline was well tolerated with the number of patients with adverse events similar to those of the control groups.

The mannitol studies both showed a significantly increased $24 \mathrm{~h}$ sputum weight after treatment compared to the control arms, consistent with improved mucociliary clearance [93, 94]. Mean $24 \mathrm{~h}$ sputum weight decreased progressively during the study in both mannitol and control arms of both studies, but remained higher in the mannitol arms throughout.

No change in lung function was observed in the studies with mannitol $[93,94]$ or hypertonic saline $6 \%$ [91]. However, a significant improvement in FEV1 and FVC was shown with hypertonic saline $7 \%$ at 3 months [92]. In contrast, a decrease in FEV1 was demonstrated in patients treated with RhDNase. 
There is insufficient evidence to permit evaluation of the use of oral mucolytics such as carbocisteine for bronchiectasis [89].

\section{Justification of the recommendation}

In summary, despite the wide heterogeneity in studies (agent used, study design and treatment duration), overall the literature showed a small improvement in the time to first exacerbation with a slightly elevated but acceptable adverse event profile with inhaled long-term mucoactive agents. The reported improvements in quality of life indicate that a proportion of patients will experience a significant benefit with these agents, but many patients will not.

The current research evidence and the ELF/EMBARC bronchiectasis patient advisory group suggest that patients give intermediate value to this treatment and acknowledge difficulties with its administration. Mucoactive therapy is time-consuming and the therapeutic equipment, in the case of nebulisers, may be difficult to take outside of the patient's home.

\section{Implementation considerations}

The indication and type of treatment given should be tailored to each individual patient according to their baseline symptom profile (frequency and severity of exacerbations, quality of life, bronchial hyperreactivity, and sputum viscosity), baseline lung function and patient preferences. We suggest testing tolerance prior to starting therapy and to consider beta-agonist premedication.

Larger studies should be considered in the future to investigate optimal treatment, dosages, durations and combinations.

\section{Question 7: Is long-term bronchodilator treatment ( $\geqslant 3$ months) compared to no treatment beneficial for adult bronchiectasis patients? \\ Recommendations}

We suggest not routinely offering long-acting bronchodilators for adult patients with bronchiectasis (conditional recommendation, very low quality of evidence)

We suggest to offer long acting bronchodilators for patients with significant breathlessness on an individual basis (weak recommendation, very low quality of evidence).

We suggest using bronchodilators before physiotherapy, inhaled mucoactive drugs, as well as before inhaled antibiotics, in order to increase tolerability and optimise pulmonary deposition in diseased areas of the lungs (good practice point, indirect evidence).

We suggest that the diagnosis of bronchiectasis should not affect the use of long acting bronchodilators in patients with comorbid asthma or COPD (good practice point, indirect evidence) [95, 96].

Where multiple inhaled therapies are used in the same patient, the sequence of treatments shown in figure 5 is commonly used by members of the task force.

\section{Summary of the evidence}

Very limited and indirect evidence is available for the benefit of the long-term treatment with bronchodilators from a systematic review that included a single trial, comparing high-dose inhaled




corticosteroids to medium-dose inhaled corticosteroid/long acting beta-agonist combination [97]. The results from this study indicate some positive effects on symptom control/symptomatic improvement, in particular decreased dyspnoea, better cough control, better health-related quality of life (measured by SGRQ symptoms domain), and reduced use of $\beta_{2}$-agonist rescue medication. Specific side-effects were generally mild (tremor, nervousness and tachycardia). A systematic review identified major methodological and reporting concerns relating to this trial [56]. However, extrapolating evidence from populations with other obstructive airway diseases some bronchiectasis subpopulations may benefit from bronchodilators, in particular subjects with chronic obstructive airflow limitation (FEV1/FVC $<0.7$; with or without FEV1 reversibility to bronchodilators), or associated asthma in combination with inhaled corticosteroids $[95,96]$.

\section{Justification of the recommendations}

We suggest the use of bronchodilators in patient with significant breathlessness due to the feasibility of application, the easy availability at a primary care level, the comparatively low treatment costs, and a putatively positive ratio of benefits to adverse events. Appropriate inhalation device selection and inhaler technique training are recommended. If treatment with bronchodilators does not result in a reduction in symptoms it should be discontinued. There is no evidence to support the use of bronchodilators routinely as part of the management of bronchiectasis patients without symptomatic breathlessness. According to both research evidence and patient advisory group feedback, it seems that patients regard this as a low risk and low burden intervention.

\section{Implementation considerations}

The intervention is easy to administer and acceptable to the majority of patients. Further investigator-driven research on the benefit of bronchodilators in bronchiectasis in various clinical situations is needed.

\section{Question 8: Are surgical interventions more beneficial compared to standard (non-surgical) treatment for adult bronchiectasis patients? \\ Recommendation}

We suggest not offering surgical treatments for adult patients with bronchiectasis with the exception of patients with localised disease and a high exacerbation frequency despite optimisation of all other aspects of their bronchiectasis management (weak recommendation, very low quality of evidence).

\section{Summary of the evidence}

The rationale for surgical treatment of bronchiectasis is to break the vicious circle of bronchiectasis by removing the lung segments that are no longer functional, and preventing the contamination of adjacent lung zones. The most frequent indication for the operation is recurrent infections with chronic symptoms such as productive cough, purulent sputum and haemoptysis [98, 99].

Lobectomy is the most frequently performed operation, but numerous options have been described (e.g. segmentectomy and pneumonectomy) [100-102]. Surgery is the procedure of choice for massive haemoptysis refractory to bronchial artery embolisation, but emergency surgery in unstable patients is associated with higher morbidity and mortality reaching 37\% [103]. Although bilateral bronchiectasis (reported in 5.8 to $30 \%$ of surgical series) are not an absolute contraindication for surgery [104], other options such as prolonged conservative treatment or bronchial artery embolisation are frequently used as an alternative. The video-assisted thoracoscopic surgery (VATS) is often preferred to better preserve lung function or reduce scarring. In comparison with open surgery, VATS has been reported to produce comparable symptomatic improvement (94 versus 88\%), but with shorter hospital stay, fewer complications (17.5 versus 23.7\%) and less pain after VATS procedures [105]. Contraindications to VATS include major parenchymal or pleural fibrosis, and calcified nodes close to the hilar vessels.

No randomised controlled trials of surgical treatment versus standard care were identified. A meta-analysis included 38 observational studies with 5541 patients, dealing with efficacy and safety of different surgical interventions for adult patients with bronchiectasis focused on three main outcomes: mortality, morbidity (adverse events) and quality of life improvement (symptomatic changes defined as reduction or alleviation of preoperative symptoms) [98].

The pooled mortality from 29 studies that focused on adult patients was $1.4 \%$ (95\% CI $0.8 \%-2.5 \%$ ) [98]. Post-operative pooled morbidity for adults was analysed in 26 observational studies and was $16.2 \%$ (95\% CI,12.5\%-19.8\%) [98]. It needs to be emphasised that there are no data comparing morbidity to continued medical non-surgical management alone. Moreover, according to the aforementioned studies, some of the morbidity is considered relatively minor (air leak, atelectasis, wound infection). Symptomatic changes were analysed in 26 observational studies. In the pooled meta-analysis, complete alleviation of symptoms was 
seen in $71.5 \%$ (95\% CI 68-74.9) and reduction of preoperative symptoms was seen in $20.2 \%$ of the adult population (95\% CI 17.3-23.1) [98]. Other research has shown that extent of residual bronchiectasis and P. aeruginosa infection were reported as unfavourable prognostic factors [99].

\section{Justification of the recommendations}

Overall, surgical interventions seem to be beneficial only in very carefully selected patients requiring the best risk-benefit profile of improved symptoms against the morbidity associated with surgery. Feedback from the ELF/EMBARC patient advisory group suggests that patients would choose surgery only if there was no effective medical option for treatment and this feedback informs the recommendation.

\section{Implementation considerations}

Involvement of an experienced surgeon in partnership with an expert respiratory physician is advisable if surgical treatment is being considered. Attention should be paid to pre-operative nutritional status and pulmonary rehabilitation. More research is needed on surgical interventions. Although a randomised trial would be very challenging future studies should include a matched control population with meticulous description of other treatments used in both populations.

\section{Question 9: Is regular physiotherapy lairway clearance and/or pulmonary rehabilitation) more beneficial than control (no physiotherapy treatment) in adult bronchiectasis patients? \\ Recommendations}

We suggest that patients with chronic productive cough or difficulty to expectorate sputum should be taught an airway clearance technique (ACT) by a trained respiratory physiotherapist to perform once or twice daily (weak recommendation, low quality of evidence).

We recommend that adult patients with bronchiectasis and impaired exercise capacity should participate in a pulmonary rehabilitation programme and take regular exercise. All interventions should be tailored to the patient's symptoms, physical capability and disease characteristics (strong recommendation, high quality of evidence).

\section{Summary of the evidence (figure 6)}

In bronchiectasis, it is a common belief that physiotherapy can improve mucus clearance and reduce lung inflammation and risk of infection. In addition, it is well accepted by patients. Respiratory physiotherapy includes ACTs and pulmonary rehabilitation [106, 107]. ACTs consist of breathing techniques, e.g. active cycle of breathing and autogenic drainage, sometimes combined with an instrument, e.g. flutter or

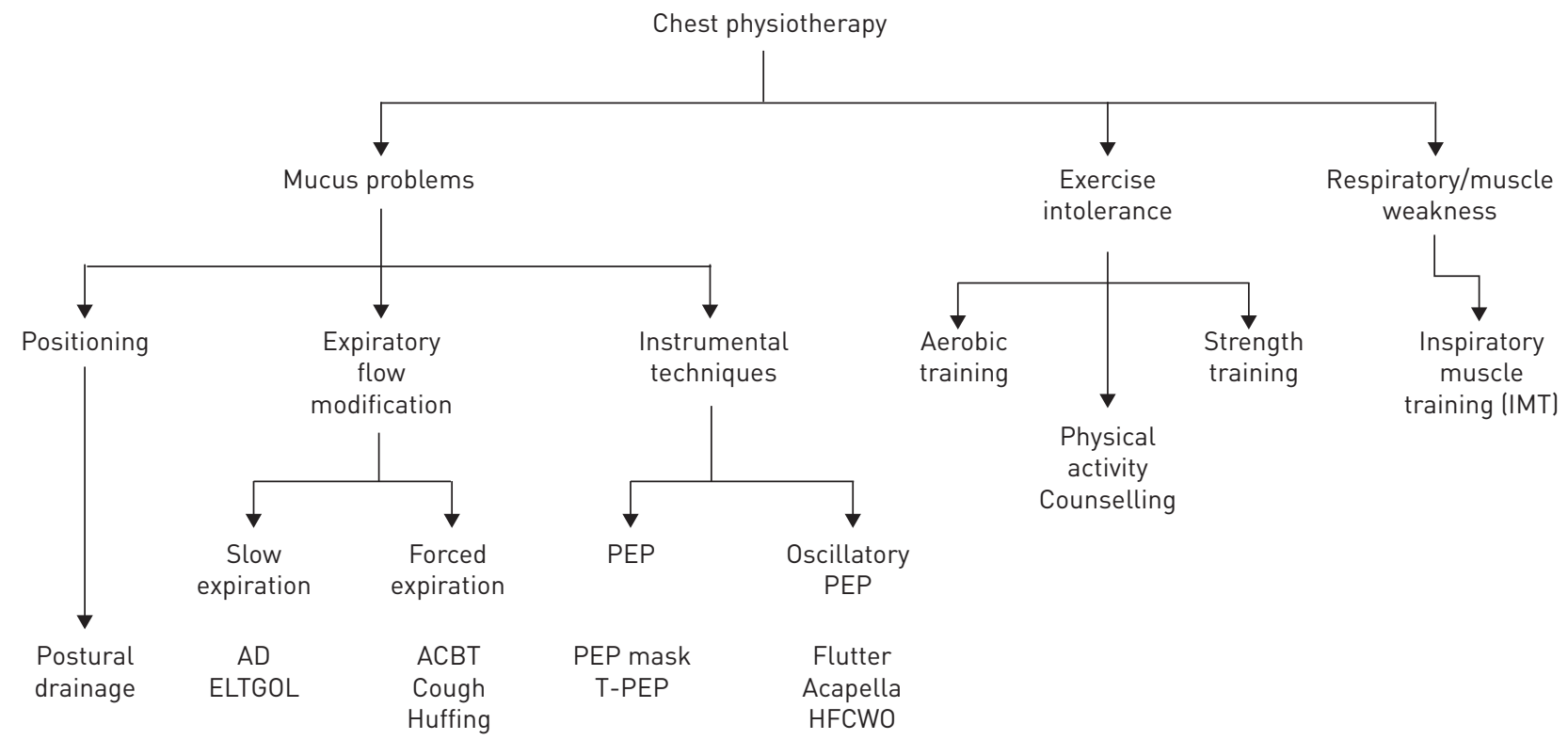

FIGURE 6 Chest physiotherapy interventions flow chart based on clinical experience from the task force panel. AD: autogenic drainage; ELTGOL: total slow expiration with open glottis and infralateral position; ACBT: active cycle of breathing techniques; PEP: positive expiratory pressure; T-PEP: temporary positive expiratory pressure; HFCWO: high frequency chest wall oscillation. 
TABLE 2 Summary of PICO questions and recommendations

\section{Question Title}

Question 1 Is standardised testing for the cause of bronchiectasis beneficial when compared with no standardised testing?

Question 2 Are courses of 14-21 days of systemic antibiotic therapy compared to shorter courses ( $<14$ days) beneficial for treating adult bronchiectasis patients with an acute exacerbation?

Question 3 Is an eradication treatment beneficial for treating bronchiectasis patients with a new isolate of a potentially pathogenic microorganism in comparison to no eradication treatment?

Question 4 Is long-term ( $\geqslant 3$ months) anti-inflammatory treatment compared to no treatment beneficial for treating adult bronchiectasis patients?

Question 5 Is long-term antibiotic treatment ( $\geqslant 3$ months) compared to no treatment beneficial for treating adult bronchiectasis patients?

\section{Recommendations}

We suggest the minimum bundle of aetiological tests in adults with a new diagnosis of bronchiectasis /conditional recommendation, very low quality of evidence) is:

1) Differential blood count

2) Serum immunoglobulins (total $\lg G, \lg A, \lg M$ )

3) Testing for allergic bronchopulmonary aspergillosis

It is expected that sputum culture is undertaken for monitoring purposes of bacterial infection. Mycobacterial culture may be helpful in selected cases where non-tuberculous mycobacteria are suspected as an aetiological cause of bronchiectasis. Additional tests may be appropriate in response to specific clinical features, or in patients with severe or rapidly progressive disease.

We suggest acute exacerbations of bronchiectasis should be treated with 14 days of antibiotics (conditional recommendation, very low quality of evidence).

It is possible that shorter or longer courses of antibiotics may be appropriate in some cases, depending on specific clinical conditions (such as exacerbation severity, patient response to treatment, or microbiology).

We suggest that adults with bronchiectasis with a new isolation of $P$. aeruginosa should be offered eradication antibiotic treatment (conditional recommendation, very low quality of evidence).

We suggest not offering eradication antibiotic treatment to adults with bronchiectasis following new isolation of pathogens other than $P$. aeruginosa (conditional recommendation, very low quality of evidencel.

We suggest not offering treatment with inhaled corticosteroids to adults with bronchiectasis (conditional recommendation, low quality of evidence).

We recommend not offering statins for the treatment of bronchiectasis (strong recommendation, low quality of evidence).

We suggest that the diagnosis of bronchiectasis should not affect the use of inhaled corticosteroids in patients with comorbid asthma or chronic obstructive pulmonary disease lbest practice advice, indirect evidence).

We suggest offering long-term antibiotic treatment for adults with bronchiectasis who have three or more exacerbations per year (conditional recommendation, moderate quality of evidence).

All subsequent recommendations refer to patients with three or more exacerbations per year.

We suggest long-term treatment with an inhaled antibiotic for adults with bronchiectasis and chronic $P$. aeruginosa infection (conditional recommendation, moderate quality of evidence).

We suggest macrolides (azithromycin, erythromycin) for adults with bronchiectasis and chronic $P$. aeruginosa infection in whom an inhaled antibiotic is contraindicated, not tolerated or not feasible (conditional recommendation, low quality of evidence).

We suggest macrolides (azithromycin, erythromycin) in addition to or in place of an inhaled antibiotic, for adults with bronchiectasis and chronic $P$. aeruginosa infection who have a high exacerbation frequency despite taking an inhaled antibiotic (conditional recommendation, low quality of evidence).

We suggest long-term macrolides (azithromycin, erythromycin) for adults with bronchiectasis not infected with $P$. aeruginosa (conditional recommendation, moderate quality of evidence).

We suggest long-term treatment with an oral antibiotic (choice based on antibiotic susceptibility and patient tolerancel for adults with bronchiectasis not infected with $P$. aeruginosa in whom macrolides are contraindicated, not tolerated or ineffective (conditional recommendation, low quality of evidence). 
TABLE 2 Continued

We suggest long-term treatment with an inhaled antibiotic for adults with bronchiectasis not infected with $P$. aeruginosa in whom oral antibiotic prophylaxis is contraindicated, not tolerated or ineffective (conditional recommendation, low quality of evidence).

Long-term antibiotic therapy should be considered only after optimisation of general aspects of bronchiectasis management (airway clearance and treating modifiable underlying causes).

Question 6 Is long-term mucoactive treatment ( $\geqslant 3$ months) compared to no treatment beneficial for treating adult bronchiectasis patients?

We suggest offering long-term mucoactive treatment ( $\geqslant 3$ months) in adult patients with bronchiectasis who have difficulty in expectorating sputum and poor quality of life and where standard airway clearance techniques have failed to control symptoms (weak recommendation, low quality of evidence).

We recommend not offering recombinant human DNase to adult patients with bronchiectasis (strong recommendation, moderate quality of evidencel.

Question 7 Is long-term bronchodilator treatment ( $\geqslant 3$ months) compared to no treatment beneficial for adult bronchiectasis patients?

We suggest not routinely offering long-acting bronchodilators for adult patients with bronchiectasis (conditional recommendation very low quality of evidence).

We suggest offering long acting bronchodilators for patients with significant breathlessness on an individual basis (weak recommendation, very low quality of evidencel.

We suggest using bronchodilators before physiotherapy, including inhaled mucoactive drugs, as well as before inhaled antibiotics, in order to increase tolerability and optimise pulmonary deposition in diseased areas of the lungs Igood practice point indirect evidence).

We suggest that the diagnosis of bronchiectasis should not affect the use of long acting bronchodilators in patients with comorbid asthma or chronic obstructive pulmonary disease (good practice point, indirect evidence) $[95,96]$.

Question 8 Are surgical interventions more beneficial compared to standard (non-surgical) treatment for adult bronchiectasis patients?

We suggest not offering surgical treatments for adult patients with bronchiectasis with the exception of patients with localised disease and a high exacerbation frequency despite optimisation of all other aspects of their bronchiectasis management (weak recommendation, very low quality of evidence).

Question 9 Is regular physiotherapy lairway clearance and/or pulmonary

We suggest that patients with chronic productive cough or difficulty to expectorate sputum should be taught an airway clearance technique by a trained respiratory physiotherapist to perform once or twice daily (weak recommendation, low quality of evidencel.

We recommend that adult patients with bronchiectasis and impaired exercise capacity should participate in a pulmonary rehabilitation programme and take regular exercise. All interventions should be tailored to the patient's symptoms, physical capability and disease characteristics (strong recommendation, high quality of evidence).

Acapella, that modify expiratory flow and volumes or produce chest wall oscillations in order to increase mucus clearance [108-112]. The principal effect obtained by ACTs is an increase in sputum volume [108, $112,113]$ and a reduced impact of cough on quality of life [109, 114]. Interesting, but still preliminary data, shows reduced peripheral airways obstruction, less inflammatory cells in sputum and improved exercise capacity after ACTs $[109,112,114]$. The aim of a pulmonary rehabilitation programme is to improve exercise tolerance and quality of life through a tailored standardised exercise protocol [115-117].

We identified three systematic reviews $[106,118,119]$ and several additional trials. We included a total of 14 clinical trials in our analysis [91, 108, 110-112, 114-117, 120-124].

The pooled analysis shows that pulmonary rehabilitation has a clear impact on exercise capacity immediately after the programme and a nonsignificant trend to improved quality of life (SGRQ) [116, 117, 
$119,122,123]$. However, the unpooled study of MANDAL et al. [122] (pulmonary rehabilitation duration (8 weeks) shorter than for pooled studies) described that improvements on exercise capacity and patient's quality of life may be maintained for a longer period of time. Finally, there is one publication showing an impact of pulmonary rehabilitation ( 8 weeks of supervised exercise training and review of ACTs) decreasing the frequency of exacerbations (median 1 (interquartile range $1-3)$ versus $2(1-3) ; p=0.012$ ) over 12-month follow-up and longer time to first exacerbation ( 8 versus 6 months; $p=0.047)$ [116]. The reported impact of both ACTs and pulmonary rehabilitation on pulmonary function is not clinically important [107-109, 112, 121].

\section{Justification of the recommendations}

The evidence for airways clearance techniques is weak because the studies are small and poorly comparable due to methodological issues. However, most studies demonstrated a significant increase of sputum volume. The evidence is stronger for pulmonary rehabilitation, showing improvements in exercise capacity, cough symptoms and quality of life, and possibly a reduction in exacerbations. The benefits of pulmonary rehabilitation are achieved in 6 to 8 weeks and maintained for between 3 to 6 months. Finally, there are no relevant adverse effects and the bronchiectasis patients advisory group value the intervention.

\section{Implementation considerations}

The research priorities in physiotherapy are: larger controlled studies with clinical outcomes (exacerbations, cough and quality of life); larger controlled studies including physiotherapy training plus mucoactive agents such as hypertonic saline; the role of pulmonary rehabilitation on exacerbations; and finally, the compliance with these interventions over a longer period of time (>12 months) [125].

\section{Summary}

The recommendations are summarised in table 2. Management of bronchiectasis aims to reduce exacerbations, reduce symptoms, improve quality of life $[126,127]$ and reduce the risk of future complications such as lung function decline [128] and severe exacerbations [129]. Treatment decisions must balance the potential beneficial effects of the intervention against the burden of treatment and the risk of adverse events. It is important to take into account the patients values and preferences in all treatment decisions, alongside the history of exacerbations, quality of life [126, 127], severity of disease [9] and underlying aetiology [22], all of which can impact on the patients long-term outcome [130-132].

The purpose of clinical guidelines is to improve the quality of patient care and to promote safe, effective and cost-effective treatment. The majority of recommendations in this guideline are conditional and based on low quality evidence. One outcome of this guideline should be to promote further research into the optimal treatment of patients with bronchiectasis. Bronchiectasis is a rapidly evolving field and our recommendations will require revision as additional data becomes available in the coming years.

\section{Acknowledgements}

The authors acknowledge Valentina Balasso for assistance with the literature search. Guideline development was supported by the European Bronchiectasis Network (EMBARC) and the EMBARC/European Lung Foundation bronchiectasis patient advisory group.

\section{References}

1 Winter DH, Manzini M, Salge JM, et al. Aging of the lungs in asymptomatic lifelong nonsmokers: findings on HRCT. Lung 2015; 193: 283-290.

2 Brozek JL, Akl EA, Compalati E, et al. Grading quality of evidence and strength of recommendations in clinical practice guidelinesPart 3 of 3 . The GRADE approach to developingrecommendations. Allergy 2011; 64: 1109-1116.

3 Andrews J, Guyatt G, Oxman AD, et al. GRADE guidelines: 14. Going from evidence to recommendations: The significance and presentation of recommendations. J Clin Epidemiol 2013; 66: 719-725.

4 King PT, Holdsworth SR, Freezer NJ, et al. Characterisation of the onset and presenting clinical features of adult bronchiectasis. Respir Med 2006; 100: 2183-2189.

5 Raghu G, King TE, Behr J, et al. Quality of life and dyspnoea in patients treated with bosentan for idiopathic pulmonary fibrosis (BUILD-1). Eur Respir J 2010; 35: 118-123.

6 Tashkin DP, Celli B, Senn S, et al. A 4-year trial of tiotropium in chronic obstructive pulmonary disease. $N$ Engl J Med 2008; 359: 1543-1554.

7 Chalmers JD, Smith MP, McHugh BJ, et al. Short- and long-term antibiotic treatment reduces airway and systemic inflammation in non-cystic fibrosis bronchiectasis. Am J Respir Crit Care Med 2012; 186: 657-665.

8 Sheehan RE, Wells AU, Copley SJ, et al. A comparison of serial computed tomography and functional change in bronchiectasis. Eur Respir J 2002; 20: 581-587.

9 Chalmers JD, Goeminne P, Aliberti S, et al. The bronchiectasis severity index. An international derivation and validation study. Am J Respir Crit Care Med 2014; 189: 576-585.

10 Aliberti S, Lonni S, Dore S, et al. Clinical phenotypes in adult patients with bronchiectasis. Eur Respir J 2016; 47: 1113-1122. 
11 Kapur N, Masters IB, Chang AB. Longitudinal growth and lung function in pediatric non-cystic fibrosis bronchiectasis: What influences lung function stability? Chest 2010; 138: 158-164.

12 Chalmers JD, Aliberti S, Polverino E, et al. The EMBARC European Bronchiectasis Registry: protocol for an international observational study. ERJ Open Res 2016; 2: 00081-2015.

13 Loebinger MR, Wells AU, Hansell DM, et al. Mortality in bronchiectasis: a long-term study assessing the factors influencing survival. Eur Respir J 2009; 34: 843-849.

14 McDonnell MJ, Aliberti S, Goeminne PC, et al. Multidimensional severity assessment in bronchiectasis: an analysis of seven European cohorts. Thorax 2016; 71: 1110-1118.

15 Finch S, McDonnell MJ, Abo-Leyah H, et al. A comprehensive analysis of the impact of Pseudomonas aeruginosa colonization on prognosis in adult bronchiectasis. Ann Am Thorac Soc 2015; 12: 1602-1611.

16 Wilson CB, Jones PW, O'Leary CJ, et al. Systemic markers of inflammation in stable bronchiectasis. Eur Respir J 1998; 12: 820-824.

17 Tsang KW, Chan KN, Ho PL, et al. Sputum elastase in steady-state bronchiectasis. Chest 2000; 117: $420-426$.

18 Chalmers JD, Moffitt KL, Suarez-Cuartin G, et al. Neutrophil elastase activity is associated with exacerbations and lung function decline in bronchiectasis. Am J Respir Crit Care Med 2017; 195: 1384-1393.

19 Chalmers JD, Hill AT. Mechanisms of immune dysfunction and bacterial persistence in non-cystic fibrosis bronchiectasis. Mol Immunol 2013; 55: 27-34.

20 Snijders D, Dominguez BF, Calgaro S, et al. Mucociliary clearance techniques for treating non-cystic fibrosis bronchiectasis: Is there evidence? Int J Immunopathol Pharmacol 2015; 28: 150-159.

21 McDonnell MJ, Aliberti S, Goeminne PC, et al. Comorbidities and the risk of mortality in patients with bronchiectasis: an international multicentre cohort study. Lancet Respir Med 2016; 4: 969-979.

22 Du Q, Jin J, Liu X, et al. Bronchiectasis as a comorbidity of chronic obstructive pulmonary disease: A systematic review and meta-analysis. PLoS One 2016; 11: e0150532.

23 Navaratnam V, Muirhead CR, Hubbard RB, et al. Critical care admission trends and outcomes in individuals with bronchiectasis in the UK. QJM 2016; 109: 523-526.

24 Navaratnam V, Millett ER, Hurst JR, et al. Bronchiectasis and the risk of cardiovascular disease: a population-based study. Thorax 2017; 72: 161-166.

25 Ringshausen FC, de Roux A, Pletz MW, et al. Bronchiectasis-associated hospitalizations in Germany, 2005-2011: a population-based study of disease burden and trends. PLoS One 2013; 8: e71109.

26 Weycker D, Edelsberg J, Oster G, et al. Prevalence and economic burden of bronchiectasis. Clin Pulm Med 2005 12: 205-209.

27 Kwak HJ, Moon J-YY, Choi YW, et al. High prevalence of bronchiectasis in adults: analysis of CT findings in a health screening program. Tohoku J Exp Med 2010; 222: 237-242.

28 Quint JK, Millett ER, Joshi M, et al. Changes in the incidence, prevalence and mortality of bronchiectasis in the UK from 2004 to 2013: A population-based cohort study. Eur Respir J 2016; 47: 186-193.

29 Chang $\mathrm{AB}$, Grimwood $\mathrm{K}$, Mulholland $\mathrm{EK}$, et al. Bronchiectasis in Indigenous children in remote Australian communities. Med J Aust 2002; 177: 200-204.

30 Seitz AE, Olivier KN, Steiner CA, et al. Trends and burden of bronchiectasis-associated hospitalizations in the United States, 1993-2006. Chest 2010; 138: 944-949.

31 Finklea JD, Khan G, Thomas S, et al. Predictors of mortality in hospitalized patients with acute exacerbation of bronchiectasis. Respir Med 2010; 104: 816-821.

32 Goeminne PC, Nawrot TS, Ruttens D, et al. Mortality in non-cystic fibrosis bronchiectasis: A prospective cohort analysis. Respir Med 2014; 108: 287-296.

33 de la Rosa D, Martinez-Garcia MA, Olveira C, et al. Annual direct medical costs of bronchiectasis treatment: Impact of severity, exacerbations, chronic bronchial colonization and chronic obstructive pulmonary disease coexistence. Chron Respir Dis 2016; 13: 361-371.

34 Gursel G. Does coexistence with bronchiectasis influence intensive care unit outcome in patients with chronic obstructive pulmonary disease? Heart Lung 2006; 35: 58-65.

35 O'Donnell AE, Barker AF, Ilowite JS, et al. Treatment of idiopathic bronchiectasis with aerosolized recombinant human DNase I. Chest 1998; 113: 1329-1334.

36 Barker AF, O'Donnell AE, Flume P, et al. Aztreonam for inhalation solution in patients with non-cystic fibrosis bronchiectasis (AIR-BX1 and AIR-BX2): Two randomised double-blind, placebo-controlled phase 3 trials. Lancet Respir Med 2014; 2: 738-749.

37 Vendrell M, de Gracia J, Olveira C, et al. Normativa SEPAR: diagnóstico y tratamiento de las bronquiectasias. Arch Bronconeumol 2008; 44: 629-640.

38 Pasteur MC, Bilton D, Hill AT, British Thoracic Society Bronchiectasis non-CF Guideline Group. British Thoracic Society guideline for non-CF bronchiectasis. Thorax 2010; 65: Suppl. 1, i1-i58.

39 Woodhead M, Blasi F, Ewig S, et al. Guidelines for the management of adult lower respiratory tract infections--full version. Clin Microbiol Infect 2011; 17: Suppl. 6, E1-59.

40 Andrews JC, Schünemann HJ, Oxman AD, et al. GRADE guidelines: 15. Going from evidence to recommendation-determinants of a recommendation's direction and strength. J Clin Epidemiol 2013; 66: 726-735.

41 Alonso-Coello P, Schünemann HJ, Moberg J, et al. GRADE Evidence to Decision (EtD) frameworks: a systematic and transparent approach to making well informed healthcare choices. 1: Introduction. BMJ 2016; 353 : i2016.

42 Anwar GA, McDonnell MJ, Worthy SA, et al. Phenotyping adults with non-cystic fibrosis bronchiectasis: A prospective observational cohort study. Respir Med 2013; 107: 1001-1007.

43 Shoemark A, Ozerovitch L, Wilson R. Aetiology in adult patients with bronchiectasis. Respir Med 2007; 101: 1163-1170.

44 Pasteur MC, Helliwell SM, Houghton SJ, et al. An investigation into causative factors in patients with bronchiectasis. Am J Respir Crit Care Med 2000; 162: 1277-1284.

45 Lonni S, Chalmers JD, Goeminne PC, et al. Etiology of non-cystic fibrosis bronchiectasis in adults and its correlation to disease severity. Ann Am Thorac Soc 2015; 12: 1764-1770.

46 Greenberger PA. When to suspect and work up allergic bronchopulmonary aspergillosis. Ann Allergy Asthma Immunol 2013; 111: 1-4. 
Knutsen AP, Bush RK, Demain JG, et al. Fungi and allergic lower respiratory tract diseases. J Allergy Clin Immunol 2012; 129: 280-291.

Griffith DE, Aksamit T, Brown-Elliott BA, et al. An official ATS/IDSA statement: Diagnosis, treatment, and prevention of nontuberculous mycobacterial diseases. Am J Respir Crit Care Med 2007; 175: 367-416.

Lucas JS, Barbato A, Collins SA, et al. European Respiratory Society guidelines for the diagnosis of primary ciliary dyskinesia. Eur Respir J 2017; 49: 1601090.

Bilton D, Henig N, Morrissey B, et al. Addition of inhaled tobramycin to ciprofloxacin for acute exacerbations of Pseudomonas aeruginosa infection in adult bronchiectasis. Chest 2006; 130: 1503-1510.

Murray MP, Turnbull K, MacQuarrie S, et al. Assessing response to treatment of exacerbations of bronchiectasis in adults. Eur Respir J 2009; 33: 312-317.

Orriols R, Roe J. Inhaled antibiotic therapy in non-cystic fibrosis patients with bronchiectasis and chronic broncial infection by Pseudomonas aeruginosa. Respir Med 1999; 93: 476-480.

McDonnell MJ, Jary HR, Perry A, et al. Non cystic fibrosis bronchiectasis: A longitudinal retrospective observational cohort study of Pseudomonas persistence and resistance. Respir Med 2015; 109: 716-726.

Orriols R, Hernando R, Ferrer A, et al. Eradication therapy against Pseudomonas aeruginosa in non-cystic fibrosis bronchiectasis. Respiration 2015; 90: 299-305.

White L, Mirrani G, Grover M, et al. Outcomes of Pseudomonas eradication therapy in patients with non-cystic fibrosis bronchiectasis. Respir Med 2012; 106: 356-360.

Goyal V, Chang AB. Combination inhaled corticosteroids and long-acting beta2-agonists for children and adults with bronchiectasis. Cochrane Database Syst Rev 2014; CD010327.

Steele K, Greenstone M, Lasserson JA. Oral methyl-xanthines for bronchiectasis. Cochrane Database Syst Rev 2001; CD002734.

Lasserson T, Holt K. Oral corticosteroids for bronchiectasis (stable and acute exacerbations). Cochrane Database Syst Rev 2001; CD002162.

Corless JA, Warburton CJ. Leukotriene receptor antagonists for non-cystic fibrosis bronchiectasis. Cochrane Database Syst Rev 2000; CD002174.

Kapur N, Chang AB. Oral non steroid anti-inflammatories for children and adults with bronchiectasis. Cochrane Database Syst Rev 2007; CD006427.

Pizzutto SJ, Upham JW, Yerkovich ST, et al. Inhaled non-steroid anti-inflammatories for children and adults with bronchiectasis. Cochrane Database Syst Rev 2016; CD007525.

Tsang KW, Tan KC, Ho PL, et al. Inhaled fluticasone in bronchiectasis: a 12 month study. Thorax 2005; 60: 239-243.

Mandal P, Chalmers JD, Graham C, et al. Atorvastatin as a stable treatment in bronchiectasis: A randomised controlled trial. Lancet Respir Med 2014; 2: 455-463.

Hernando R, Drobnic ME, Cruz MJ, et al. Budesonide efficacy and safety in patients with bronchiectasis not due to cystic fibrosis. Int J Clin Pharm 2012; 34: 644-650.

Brodt AM, Stovold E, Zhang L. Inhaled antibiotics for stable non-cystic fibrosis bronchiectasis: a systematic review. Eur Respir J 2014; 44: 382-393. systematic review and meta-analysis. PLoS One 2014; 9: e90047.

macrolides in bronchiectasis patients: a meta-analysis and systematic review. Pulm Pharmacol Ther 2014; 28: 171-178.

Wu Q, Shen W, Cheng H, et al. Long-term macrolides for non-cystic fibrosis bronchiectasis: A systematic review and meta-analysis. Respirology 2014; 19: 321-329.

Zhuo GY, He Q, Xiang-Lian L, et al. Prolonged treatment with macrolides in adult patients with non-cystic fibrosis bronchiectasis: Meta-analysis of randomized controlled trials. Pulm Pharmacol Ther 2014; 30: 1-9.

Fan LC, Lu HW, Wei P, et al. Effects of long-term use of macrolides in patients with non-cystic fibrosis bronchiectasis: a meta-analysis of randomized controlled trials. BMC Infect Dis 2015; 15: 160.

Yang JW, Fan LC, Lu HW, et al. Efficacy and safety of long-term inhaled antibiotic for patients with noncystic fibrosis bronchiectasis: a meta-analysis. Clin Respir J 2016; 10: 731-739.

Hnin K, Nguyen C, Carson K V, et al. Prolonged antibiotics for non-cystic fibrosis bronchiectasis in children and adults. Cochrane Database Syst Rev 2015; 8: CD001392.

Currie DC, Garbett ND, Chan KL, et al. Double-blind randomized study of prolonged higher-dose oral amoxycillin in purulent bronchiectasis. Q J Med 1990; 76: 799-816.

Cymbala AA, Edmonds LC, Bauer MA, et al. The disease-modifying effects of twice-weekly oral azithromycin in patients with bronchiectasis. Treat Respir Med 2005; 4: 117-122.

Drobnic ME, Suñé P, Montoro JB, et al. Inhaled tobramycin in non-cystic fibrosis patients with bronchiectasis and chronic bronchial infection with Pseudomonas aeruginosa. Ann Pharmacother 2005; 39: 39-44. fibrosis bronchiectasis. Am J Respir Crit Care Med 2011; 183: 491-499.

C, ayaram L, Karalus N, et al. Azithromycin for prevention of exacerbations in non-cystic fi brosis bronchiectasis (EMBRACE): A randomised, double-blind, placebo-controlled trial. Lancet 2012; 380: 660-667.

Serisier DJ, Bilton D, De Soyza A, et al. Inhaled, dual release liposomal ciprofloxacin in non-cystic fibrosis bronchiectasis (ORBIT-2): a randomised, double-blind, placebo-controlled trial. Thorax 2013; 68: 812-817.

Serisier DJ, Martin ML, McGuckin MA, et al. Effect of long-term, low-dose erythromycin on pulmonary exacerbations among patients with non-cystic fibrosis bronchiectasis: the BLESS randomized controlled trial. JAMA 2013; 309: 1260-1267.

Altenburg J. Effect of azithromycin maintenance treatment on infectious exacerbations among patients with non-cystic fibrosis bronchiectasis. JAMA 2013; 309: 1251-1259.

De Diego A, Milara J, Martinez-Moragón E, et al. Effects of long-term azithromycin therapy on airway oxidative stress markers in non-cystic fibrosis bronchiectasis. Respirology 2013; 18: 1056-1062.

Tabernero Huguet E, Gil Alaña P, Alkiza Basañez R, et al. Inhaled colistin in elderly patients with non-cystic fibrosis bronchiectasis and chronic Pseudomonas aeruginosa bronchial infection. Rev española Geriatr y Gerontol 2015; 50: 111-115. 
83 Haworth CS, Foweraker JE, Wilkinson P, et al. Inhaled colistin in patients with bronchiectasis and chronic Pseudomonas aeruginosa infection. Am J Respir Crit Care Med 2014; 189: 975-982

84 PROLONGED antibiotic treatment of severe bronchiectasis; a report by a subcommittee of the Antibiotics Clinical Trials (non-tuberculous) Committee of the Medical Research Council. Br Med J 1957; 2: 255-259.

85 Lourdesamy AI, Muthukumaru U. Efficacy of azithromycin in the treatment of bronchiectasis. Respirology 2014; 19: $1178-1182$.

86 Liu J, Zhong X, He Z, et al. Effect of Low-Dose, long-term roxithromycin on airway inflammation and remodeling of stable noncystic fibrosis bronchiectasis. Mediators Inflamm 2014; 2014: 708608.

87 Barker AF, Couch L, Fiel SB, et al. Tobramycin solution for inhalation reduces sputum Pseudomonas aeruginosa density in bronchiectasis. Am J Respir Crit Care Med 2000; 162: 481-485.

88 Hart A, Sugumar K, Milan SJ, et al. Inhaled hyperosmolar agents for bronchiectasis. Cochrane Database Syst Rev 2014; 5: CD002996.

89 Wilkinson M, Sugumar K, Milan SJ, et al. Mucolytics for bronchiectasis. Cochrane Database Syst Rev 2014; 5 : CD001289.

90 Wills P, Greenstone M. Inhaled hyperosmolar agents for bronchiectasis. Cochrane Database Syst Rev 2002; 1: CD002996.

91 Nicolson CHH, Stirling RG, Borg BM, et al. The long term effect of inhaled hypertonic saline 6\% in non-cystic fibrosis bronchiectasis. Respir Med 2012; 106: 661-667.

92 Kellett F, Robert NM. Nebulised 7\% hypertonic saline improves lung function and quality of life in bronchiectasis. Respir Med 2011; 105: 1831-1835.

93 Bilton D, Daviskas E, Anderson D, et al. Phase 3 randomized study of the efficacy and safety of inhaled dry powder mannitol for the symptomatic treatment of non-cystic fibrosis bronchiectasis. Chest 2013; 144: 215-225.

94 Bilton D, Tino G, Barker AF, et al. Inhaled mannitol for non-cystic fibrosis bronchiectasis: a randomised, controlled trial. Thorax 2014; 69: 1073-1079.

95 Vogelmeier CF, Criner GJ, Martinez FJ, et al. Global Strategy for the Diagnosis, Management, and Prevention of Chronic Obstructive Lung Disease 2017 Report: GOLD Executive Summary. Eur Respir J 2017; 49: 1700214.

96 Reddel HK, Bateman ED, Becker A, et al. A summary of the new GINA strategy: A roadmap to asthma control. Eur Respir J 2015; 46: 622-639.

97 Martínez-García MÁ, Soler-Cataluña JJ, Catalán-Serra P, et al. Clinical efficacy and safety of budesonide-formoterol in non-cystic fibrosis bronchiectasis. Chest 2012; 141: 461-468.

98 Fan L-C, Liang S, Lu H-W, et al. Efficiency and safety of surgical intervention to patients with non-cystic fibrosis bronchiectasis: a meta-analysis. Sci Rep 2015; 5: 17382.

99 Hiramatsu M, Shiraishi Y, Nakajima Y, et al. Risk factors that affect the surgical outcome in the management of focal bronchiectasis in a developed country. Ann Thorac Surg 2012; 93: 245-250.

100 Hoffman E. The late results of the conservation of the apical segment of the lower lobe in resections for bronchiectasis. Thorax 1955; 10: 137-141.

101 Collis J. Fate of the lower apical segment in resections for bronchiectasis. Thorax 1953; 8: 323-325.

102 Tanaka H, Matsumura A, Okumura M, et al. Pneumonectomy for unilateral destroyed lung with pulmonary hypertension due to systemic blood flow through broncho-pulmonary shunts. Eur J Cardiothoracic Surg 2005; 28 : 389-393.

103 Gourin A, Garzon AA. Operative treatment of massive hemoptysis. Ann Thorac Surg 1974; 18: 52-60.

104 Schneiter D, Meyer N, Lardinois D, et al. Surgery for non-localized bronchiectasis. Br J Surg 2005; 92: 836-839.

105 Zhang P, Zhang F, Jiang S, et al. Video-assisted thoracic surgery for bronchiectasis. Ann Thorac Surg 2011; 91: 239-243.

106 Lee AL, Burge AT, Holland AE. Airway clearance techniques for bronchiectasis. Cochrane database Syst Rev 2015; 11: CD008351.

107 Bradley J, Moran F, Greenstone M. Physical training for bronchiectasis. Cochrane Database Syst Rev 2002; CD002166.

108 Guimarães FS, Moço VJR, Menezes SLS, et al. Effects of ELTGOL and Flutter VRP1 ${ }^{\circledR}$ on the dynamic and static pulmonary volumes and on the secretion clearance of patients with bronchiectasis. Rev Bras Fisioter 2012; 16: $108-113$.

109 Nicolini A, Cardini F, Landucci N, et al. Effectiveness of treatment with high-frequency chest wall oscillation in patients with bronchiectasis. BMC Pulm Med 2013; 13: 21.

110 Eaton T, Young P, Zeng I, et al. A randomized evaluation of the acute efficacy, acceptability and tolerability of flutter and active cycle of breathing with and without postural drainage in non-cystic fibrosis bronchiectasis. Chron Respir Dis 2007; 4: 23-30.

111 Naraparaju S, Vaishali K, Venkatesan P, et al. A comparison of the Acapella and a threshold inspiratory muscle trainer for sputum clearance in bronchiectasis-A pilot study. Physiother Theory Pract 2010; 26: 353-357.

112 Figueiredo PHS, Zin WA, Guimarães FS. Flutter valve improves respiratory mechanics and sputum production in patients with bronchiectasis. Physiother Res Int 2012; 17: 12-20.

113 Patterson JE, Bradley JM, Hewitt O, et al. Airway clearance in bronchiectasis: A randomized crossover trial of active cycle of breathing techniques versus acapella? Respiration 2005; 72: 239-242.

114 Murray MP, Pentland JL, Hill AT. A randomised crossover trial of chest physiotherapy in non-cystic fibrosis bronchiectasis. Eur Respir J 2009; 34: 1086-1092.

115 Faulkner J, Gerhard J, Stoner L, et al. Self-paced walking within a diverse topographical environment elicits an appropriate training stimulus for cardiac rehabilitation patients. Rehabil Res Pract 2012; 2012: 140871

116 Lee AL, Hill CJ, Cecins N, et al. The short and long term effects of exercise training in non-cystic fibrosis bronchiectasis - a randomised controlled trial. Respir Res 2014; 15: 44.

117 Zanini A, Aiello M, Adamo D, et al. Effects of pulmonary rehabilitation in patients with non-cystic fibrosis bronchiectasis: a retrospective analysis of clinical and functional predictors of efficacy. Respiration 2015; 89: 525-533.

118 Bradley J, Moran F, Greenstone M. Physical training for bronchiectasis. Cochrane Database Syst Rev 2002; CD002166. 
119 Lee AL, Hill CJ, McDonald CF, et al. Pulmonary rehabilitation in individuals with non-cystic fibrosis bronchiectasis: a systematic review. Arch Phys Med Rehabil 2016; 774-782.e1.

120 Patterson JE, Bradley JM, Elborn JS. Airway clearance in bronchiectasis: a randomized crossover trial of active cycle of breathing techniques (incorporating postural drainage and vibration) versus test of incremental respiratory endurance. Chron Respir Dis 2004; 1: 127-130.

121 Gokdemir Y, Karadag-Saygi E, Erdem E, et al. Comparison of conventional chest physiotherapy and high-frequency chest wall oscillation in primary ciliary dyskinesia. Pediatr Pulmonol 2014; 49: 611-616.

122 Mandal P, Sidhu MK, Kope L, et al. A pilot study of pulmonary rehabilitation and chest physiotherapy versus chest physiotherapy alone in bronchiectasis. Respir Med 2012; 106: 1647-1654.

123 Newall C, Stockley RA, Hill SL. Exercise training and inspiratory muscle training in patients with bronchiectasis. Thorax 2005; 60: 943-948.

124 Liaw M-Y, Wang Y-H, Tsai Y-C, et al. Inspiratory muscle training in bronchiectasis patients: a prospective randomized controlled study. Clin Rehabil 2011; 25: 524-536.

125 Aliberti S, Masefield S, Polverino E, et al. Research priorities in bronchiectasis: a consensus statement from the EMBARC Clinical Research Collaboration. Eur Respir J 2016; 48: 632-647.

126 Wilson CB, Jones PW, O'Leary CJ, et al. Validation of the St. George's respiratory questionnaire in bronchiectasis. Am J Respir Crit Care Med 1997; 156: 536-541.

127 Quittner AL, O'Donnell AE, Salathe MA, et al. Quality of Life Questionnaire-Bronchiectasis: final psychometric analyses and determination of minimal important difference scores. Thorax 2015; 70: 12-20.

128 Martínez-García MA, Soler-Cataluña J-J, Perpiñá-Tordera M, et al. Factors associated with lung function decline in adult patients with stable non-cystic fibrosis bronchiectasis. Chest 2007; 132: 1565-1572.

129 Chalmers JD, Aliberti S, Blasi F. Management of bronchiectasis in adults. Eur Respir J 2015; 45: 1446-1462.

130 Goeminne PC, Scheers H, Decraene A, et al. Risk factors for morbidity and death in non-cystic fibrosis bronchiectasis: a retrospective cross-sectional analysis of CT diagnosed bronchiectatic patients. Respir Res 2012; 13: 21.

131 Hill AT, Haworth CS, Aliberti S, et al. Pulmonary exacerbation in adults with bronchiectasis: a consensus definition for clinical research. Eur Respir J 2017; 49: 1700051.

132 Spinou A, Siegert RJ, Guan WJ, et al. The development and validation of the bronchiectasis health questionnaire. Eur Respir J 2017; 49: 1601532. 